University of Zurich

Department of Economics

Working Paper Series

ISSN 1664-7041 (print)

ISSN 1664-705X (online)

Working Paper No. 237

\title{
Distributional Comparative Statics with Heterogeneous Agents
}

Andreas Hefti

November 2016 


\title{
DISTRIBUTIONAL COMPARATIVE STATICS WITH HETEROGENEOUS AGENTS
}

\author{
Andreas Hefti*
}

November 22, 2016

\begin{abstract}
We propose a formal way to systematically study the differential effects of exogenous shocks in economic models with heterogeneous agents. Our setting applies to models that can be rephrased as "competition for market shares" in a broad sense. We show that even in presence of any number of arbitrarily heterogeneous agents, a single recursion relation characterizes the distributional pattern of equilibrium market shares and related measures. We identify the general conditions under which the market share function rotates, thereby either causing more or less equality among the agents. Our setting highlights the exceptional rule that power functions play for the distributional effects. We apply our method across economic models, including examples from monopolistic competition, discrete choice, partial and general equilibrium theory and contest theory.
\end{abstract}

JEL Classification: D72; D82; C73; C62; C65

Keywords: Heterogeneity; Inequality; Monopolistic Competition; General Equilibrium; Contest Theory

\section{First Version}

\footnotetext{
${ }^{*}$ Corresponding author: Andreas Hefti, Bluemlisalpstrasse 10, CH-8006 Zurich, andreas.hefti@econ.uzh.ch. Author affiliations: Department of Economics, University of Zurich and School of Management and Law, Zurich University of Applied Sciences. I wish to thank Armin Schmutzler, David Wettstein and Shuo Liu as well as the participants of the Swiss-Basel Theory Meeting 2014, EEA 2014 and UECE Lisbon Game Theory Meeting 2015 for many valuable comments.
} 


\section{Introduction}

Heterogeneous agents are omnipresent in reality. Firms have different production technologies at their disposal, workers have different abilities or skills, and consumers have different preferences or income. In contrast, economic models frequently neglect heterogeneity, either by assuming perfect symmetry among the agents, by resorting to a single representative agent, ${ }^{1}$ or by focusing more on some aggregate and less on the distributional impact of an exogenous shock. Indeed, several papers allow for heterogeneous agents when establishing existence or uniqueness of equilibrium in a given model, but resort to the simplifying symmetric case when analyzing the comparative-statics. This is well understandable given that heterogeneity typically makes a model far less tractable, particularly if one does not desire to introduce (ex ante) heterogeneity in a utterly restrictive way (e.g., only two different production technologies). The cost of such a procedure is that distributional comparative statics cannot be studied. There are many examples, where distributional concerns matter. For instance, a regulating authority would like to know which aspects of a market tend to favor a strong market concentration, or which intervention (taxes, subsidies, emission permits,...) has what distributional consequences. A sports tournament designer may want to know which prize structure makes competition most unpredictable by equalizing winning chances between stronger and weaker agents.

In this paper we seek to resolve the problem of analyzing the comparative-statics with heterogeneous agents at the general level. To this end, we develop a systematic approach to study models with payoff functions that can be formulated as "competition for market shares" in a broad way. Examples that fit this type of structure are ubiquitous in competition theory, general equilibrium theory, monopolistic competition, political economics and game theory. In general, we consider a setting where agents differ in their type (production costs, preferences, disposable income, ability,...). The problem is set up such that agents directly choose their respective equilibrium market shares. ${ }^{2}$ The respective equilibrium market share function, a density on the agent type space, is the main object of our interest. It qualitatively reflects the ex-ante heterogeneity of the agents in such that comparably "strong" agents achieve larger equilibrium market shares. An exogenous shock to the model, such as a common demand or innovation

\footnotetext{
${ }^{1}$ There are various definitions of what a representative agent could and should mean, and in many cases there is no respective representative agent (see Jackson and Yariv (2016) for a recent discussion).

${ }^{2}$ Our equilibrium concept is formally defined in section 2.1, and encompasses the standard cases of (Walrasian) equilibria (price-taking agents), monopolistic competition equilibria (with price-aggregation), aggregate-taking behavior equilibria in general, and Nash equilibrium.
} 
shock in monopolistic competition, introduction or change of a sale tax in competitive markets or introduction or change of a second winning prize in a contest, may distort the market share function. Under which circumstances does a change in the economic environment make already strong agents even stronger, and when can we expect to see a reduction of market concentration? The formal contribution of our paper is to develop a systematic strategy to study how the equilibrium market share function, and related measures such as the distribution of payoffs, efforts, quantities or similar, depend on properties or parameters of the respective model. The major insight is that we can learn much about the possible rotations of the market share function, for an arbitrary degree of agent heterogeneity, from a single optimality equation, without the need to explicitly solve for the full system of equilibrium equations, which generally is impossible.

Main results We show that the possible rotational pattern of the market share function in equilibrium is described by a recursive relation between agent types. This recursion decomposes the relation between the market shares of different types into a direct-aggregative and an indirect effect of the shock, where the former is decisive for the type of rotation, and the latter influences certain quantitative aspects of the rotation. The direct-aggregative effect captures essentially how marginal benefits respond to the shock. As a rule of thumb, we find that if marginal benefits increase more (less) than proportional for stronger agents, this tends to increase (decrease) inequality of the equilibrium market shares. Thus, if the shock hits all agents uniformly, this increases equality across agents, while shocks that affect the incentives of all agents in a proportional way have no distributional effects.

Our general results identify the remarkable role that power functions play in determining the distributional comparative statics. If (marginal) costs and benefits associated with attaining a certain market share are type-invariant power functions of the market share, then the possible rotations induced by a common shock satisfy a global transitivity condition. This follows because relative market shares of different agent types respond monotonically to such shocks, a property which we call the monotone ratio condition. Under this condition, market shares follow a particularly pronounced type of rotation, where the relative change in market share is strictly increasing in agent type. It follows that in such a case the strongest (weakest) agents gain (lose) most. Such power functions are pervasive in economic models. Examples range from monopolistic competition with CES-demand, competitive production with homogeneous 
production technology to imperfectly discriminatory contests with Tullock success function. All these models involve such power functions and thus yield strong distributional comparative-static predictions.

We apply our methods to study the distributional comparative-statics in a number of examples from different economic subfields. In case of monopolistic competition with CES-demand with different quality levels and homogeneous production technology, we show that the stronger competition resulting from more substitutable products lets already rich firm expand their market size while small firms are marginalized. We also study the more challenging case where firms have heterogeneous production elasticities. Contrary to the homogeneous case, growing income yields quantity growth jointly with a growing inequality of firm market shares. Increasing efficiency has a similar effect, while it necessarily generates winners and losers in terms of absolute profits. This occurs because large firms face less downward pressure on prices as efficiency increases while they can expand their quantities by relatively more. It follows that with spillover process innovations, the most dominant firms also have the strongest incentive to innovate, while laggards are hurt by such innovations.

In the Logit model of product competition choice we show that if the deterministic part of utility becomes more decisive (choice is less noisy) this is associated with an increasing inequality of the supplier market shares. Moreover, the results suggest that the weakest firms (in terms of low product quality or high supply costs) have the strongest incentive to increase the noisiness in the choice procedure e.g., by resorting to obfuscation tactics. In an extension we discuss the distributional effects of an import tax. We find that all domestic firms benefit from the import tax in a way that leaves relative market shares and relative profits of domestic firms unaltered. Domestic prices remain unaffected, while foreign prices increase uniformly in the tax. Moreover, if importers are weak compared to domestic suppliers, we would expect to observe attempts of importers to blur or complicate consumer perception of the market.

We also consider general equilibrium models with price-taking behavior. With price-taking firms, technology alone is decisive for the distributional comparative-static patterns. If all firms have access to a similar decreasing-returns technology, then equilibrium market shares, relative profits and relative quantities all are invariant to upward shifts of market demand, but absolute profit and quantity differences increase between strong and weak firms. However, if firm heterogeneity originates from different returns to scale, then an upward demand shift increases the 
inequality of market shares. On the contrary, market shares become more equal with exponential costs if market demand shifts up.

This analysis carries over to the case of a partial equilibrium model, where consumers sell their resources to firms and acquire the produced consumption good on a competitive market. We show that an introduction (or increase) of a quantity tax has differential implications for firmside market shares only if costs are not a common-elastic power function. For example, if firm heterogeneity is driven by different elasticities of marginal costs, then the tax increases equality of market shares on the supply side (a subsidy would have opposite effects). A similar result applies for the distribution of consumption over consumers. The tax will affect consumer-side market share only if the utility functions for the good are not common-elastic power functions. With Log-utility, the tax reduces consumption inequality of the good among consumers. A common efficiency shock on the firm side has an effect similar to a subsidy, and therefore tends to increase consumption inequality.

We further study the effects of a uniform contraction of the available production resources for the distribution of firm market shares and consumer consumption in a competitive single inputoutput private ownership economy, where income is composed of labor and capital earnings. We find that if the source of income inequality is essentially the resource endowment, then consumption inequality always increases as the resource is depleted. If however income inequality originates from the share distribution, then more or less consumption inequality can result depending on (aggregate) production possibilities. Further, we analyze a competitive private ownership economy, where a consumption output is produced by firms using only labor as input, and consumers must decide on their amount of labor to supply. We ask how the distribution of consumption and leisure across consumers depends on the state of technology in the economy and on the importance of leisure relative to consumption. In case of the former, a common positive technology shock, in terms of more efficient labor, boosts consumption, real wages and profits. As profits and wages increase proportionally, the incentives to work more and benefit from the higher wage or to rather enjoy more leisure and finance consumption from the higher capital income counterbalance each other, making labor supply and consumption shares invariant to technology. However, absolute consumption differences between the wealthy and the poor increases. If consumption becomes more important relative to leisure, consumers tend to supply more labor, increasing firm profits. This benefits capital owners more, and therefore 
consumption-side market shares become less equally distributed. It may even happen that in equilibrium the poorest end up with a lower consumption level, despite a higher propensity to consume more.

Many real-world competitions, such as obtaining a research grant, lobbying, elections, sports tournaments or advertising for market shares can best be described by means of a contest for a scarce resource. Therefore, we apply our methods to the case of (imperfectly discriminatory) contests with possibly effort-depending prize values, nesting the standard case of a (generalized) Tullock contest. In case of a common fixed prize, the distributional results logically parallel those from quantity competition. For example, we find that a prize increase favors strong contestants in terms of success chances, provided that the cost elasticities of efforts increases across agents. In the special case of the Tullock contest, we show that an increase in contest noise, i.e., a reduction in the ability to influence own success chances, always levels the playing field by equalizing winning odds. We also study the distributional consequences of an effortdepending prize function. The leading example is advertising for market shares, where ads influence consumer attention as well as the willingness to pay of attentive consumers. According to the model, we should observe increasing market concentration if advertising has persuasive or attaching effects, while market shares become more uniformly distributed if ads are more of a disturbing nature. The results from this section have some implications for tournament design, essentially because of an efficiency-equity trade-off. Letting the prize function become more sensitive to the winner's effort tends to make success chances more unequal, while aggregate effort increases. If the goal is to make the competition less predictable, which is a central design aspect of sports tournaments, then a "handicapping" of winning efforts can achieve this goal, but comes at the costs of a reduction in total efforts. As a final variation we show that introducing (or increasing) a second prize in a contest tends to make the chances of winning the first prize (likewise of winning any prize) more equal. Particularly, an even split of an available prize budget across two prizes equalizes success chances most, at the cost of minimizing total effort.

Article structure The remainder of the article is structured as follows. We outline the general model in section 2, where we introduce our main assumptions, the definition of equilibrium, and establish existence of a unique equilibrium (Theorem 1), where all agents receive some market share, and the equilibrium market share ranking is consistent with the (ex ante) ranking of 
the heterogeneous agents. In section 3 we define the concept of a rotation of the market share function, develop the essential analytical tools to study such rotations and derive some general insights about the possible causes of rotations. The main results on existence and type of rotation are presented in sections 3.2.1 and 3.2.2 (Theorems $2-4$ ). The methods and results developed there are then systematically applied in section 4 to analyze the distributional comparative statics in a number of applications across economic fields. All proofs are in the appendix.

\section{The model}

Let $I$ be an index set and $i \in I$ an economic agent. In many economic models, the payoff of each agent $i$ can be decomposed as

$$
\Pi(i)=\text { Market share }(i) * \text { Market value }(i)-\operatorname{Costs}(i)
$$

In a given model, an agent typically needs to choose a variable (or a set of variables) with the goal of maximizing (1), possibly subject to a number of (feasibility) constraints. Specifically, let

$$
\Pi(i)=\max _{t(i) \geq 0} p(i, t(i), T ; x) V(i, t(i), T ; x)-\Phi(i, t(i) ; x)
$$

where $t(i) \in \mathbb{R}$ is agent $i$ 's choice variable (depending on the application this could be a price, a quantity, an effort,...), and $T=\int_{i} t(i)$ is its aggregate. Further, $p(i)=p(i, t(i), T ; x)$ is $i$ 's market share, $V(i)=V(i, t(i), T ; x)$ the market value and $\Phi(i, t(i))$ the costs of agent $i$ given her choice of $t(i)$ and the aggregate $T$. Finally, $x \in X$ is an exogenous parameter, which influences at least one of the component functions of $\Pi(i)$.

This paper seeks to analyze how the equilibrium distribution of market shares $p(i), i \in I$, and related quantities such as $\Pi(i)$ or $t(i)$, depend on $x$ in presence of heterogeneous agents. We first need to be precise, of course, what we mean by equilibrium, and how heterogeneity is introduced into the model, which are the topics of section 2.1. Examples that fit with (1'), and are subsequently analyzed by the methods developed in this paper, are i) (Walrasian) quantity competition, monopolistic competition, discrete choice (competition with logit demand), labor provision, advertising intensity and (rent-seeking) competition in contests. 


\subsection{Heterogeneity and Equilibrium: Definition, Existence and Uniqueness}

To solve our model we use two simplifying conventions. First, we reformulate the model as a direct competition for market shares. Second, we introduce continuum agents. Both adjustments are without loss of generality with respect to the purpose of this paper.

Market shares First, it is convenient to reformulate the optimization problem (1') as

$$
\Pi(i)=\max _{p(i) \geq 0} p(i) V(i, p(i), T ; x)-\Phi(i, p(i), T ; x) .
$$

In (2) the agent directly chooses her market share $p(i)$, instead of indirectly over $t(i)$. Such a transformation is possible if the functions $p(\cdot), V(\cdot)$ and $\Phi(\cdot)$ are bijective in $t(i)$, which we always assume. ${ }^{3}$ Thus the way we solve for the equilibrium is different from how the agents truly act in the model. Working with the transformed model is useful for our comparative-static purposes, but it is more natural to think that agents directly choose certain variables (efforts, prices, quantities) that determine their respective market shares when forming an intuition.

Continuum agents Second, we set $I=[0,1]$ for the agent population. The formal advantage of working with continuum agents is that they will allow for a simpler representation of equilibrium objects. Most importantly, the market share $p(i)$ will be a (density) function $p:[0,1] \rightarrow \mathbb{R}_{+}$, rather than a discrete mapping, that changes its support as the number of agents change. Importantly, continuum agents are without loss of generality in our setting. It is possible to identify the equilibrium market share $p^{d}(i)$ for any given number of atomistic agents $n \in \mathbb{N}$ by a corresponding equilibrium density $p(i)$ with support $[0,1]$ by means of rescaling with the factor $1 / n$ (see appendix A.1 for details). For example, if $n=3$ and $p^{d}(1)=1 / 2$, $p^{d}(2)=1 / 3, p^{d}(3)=1 / 6$, then $p(i)=3 / 2, i \in[0,1 / 3), p(i)=1, i \in[1 / 3,2 / 3)$ and $p(i)=1 / 2$, $i \in[2 / 3,1]$, and $\int p(i) d i=1 / 3(3 / 2+1+1 / 2)=1$. Moreover, our formulation encompasses the case of "true" continuum agents, where the equilibrium density is a strictly monotonic, continuous function $p(i)$. While there is no direct atomistic-agent analogue to this case, we argue in appendix A.1 that this can be seen as the limiting case of a large number of distinct atomistic agents.

\footnotetext{
${ }^{3}$ We are slightly abusing notion here. If $p(i)=p(i, t(i), T ; x)$ then, assuming invertibility, $t(i)=h(i, p(i), T ; x)$ and plugging this, e.g., into the market share function $V(i, t(i), T ; x)$ would yield a new function $\hat{V}(i, p(i), T ; x)$ which, for simplicity, we again label with the function symbol $V$.
} 


\subsubsection{Equilibrium Definition}

We are now ready to formally state the definition of an equilibrium. We consider the slightly more general payoff function ${ }^{4}$

$$
\Pi(i)=B(i, p(i), T)-\Phi(i, p(i), T) .
$$

Definition 1 (Equilibrium) An equilibrium is a bounded function $p:[0,1] \rightarrow \mathbb{R}_{+}$and a number $T \in(0, \infty)$ such that
i) For each $i \in[0,1], p(i)$ solves $\max _{p(i) \geq 0} \Pi(i)$, where $\Pi(i)$ is given by (3).
ii) $\int_{0}^{1} p(i) d i=1$

An intuitive interpretation of the equilibrium conditions is that in any equilibrium each agent is choosing her action variable $t(i)$ to maximize her payoff while holding a (correct) belief about the aggregate action $T$. We will see in section 4 that this equilibrium definition identifies, e.g., Walrasian (price) equilibria, the monopolistic equilibrium with CES-utility (or logit) consumers and, with an appropriate modification, the Nash equilibrium in games with a sum-aggregative representation of payoffs (such as certain contests, or the Cournot model). We next show that under suitable technical assumptions on the benefit $(B(\cdot))$ and cost $(C(\cdot))$ functions, the above notion of equilibrium is well-defined.

\subsubsection{Heterogeneity and main assumptions}

Let $g(i, p(i), T) \equiv \frac{\partial B(\cdot)}{\partial p(i)}$ and $\varphi(i, p(i), T) \equiv \frac{\partial \Phi(\cdot)}{\partial p(i)}$ denote the marginal revenue and marginal costs, respectively. Note that, for given $T>0$, the FOC pertaining to maximizing (3) at an interior point $p(i)>0$ is

$$
g(i, p(i), T)=\varphi(i, p(i), T)
$$

Our formal analysis will be squarely centered around this innocent-looking expression. If not mentioned otherwise we will take the following assumption as satisfied.

Assumption 1 Let $\Pi(i)$ be given by (3).

\footnotetext{
$X$.

${ }^{4}$ We ignore the parameter $x$ here, having in mind that $x$ can take on any fixed value in a parameter interval
} 
(A1) For any $T>0, i \in[0,1]$ and $p(i) \geq 0$ : $\Pi(i)$ is a $C^{2}$-function of $p(i)$ and strongly quasiconcave in $p(i), g(i, 0, T)>0$ and $g(i, \cdot, T)$ is bounded from above, $\varphi(i, 0, T)=0$, $\frac{\partial \varphi(i, p, T)}{\partial p}>0$ and $\lim _{p \rightarrow \infty} \varphi(i, p, T)=\infty$.

(A2) For any $i \in[0,1]: g(i, p, \cdot)$ and $\varphi(i, p, \cdot)$ are $C^{1}$-functions, $g(i, 1,0)>0, g(i, 1, \cdot)$ is bounded from above, $\varphi(i, p, 0)=0, \varphi(i, p, \cdot)$ is strictly increasing and $\lim _{T \rightarrow \infty} \varphi(i, p, T)=\infty$ whenever $p>0$, and $g_{T}(i, p, T)<\varphi_{T}(i, p, T)$ whenever $g(i, p, T)=\varphi(i, p, T)$.

(A3) For any $p, T>0: B(\cdot, p, T), g(\cdot, p, T)$ are (weakly) decreasing and $\Phi(\cdot, p, T), \varphi(\cdot, p, T)$ (weakly) increasing on $[0,1]$.

We also maintain, for simplicity, that inaction is possible by setting $B(i, 0, T)=\Phi(i, 0, T)=0 .{ }^{5}$ Assumptions (A1) and (A2) amount to natural differentiability, boundary and slope assumptions. In particular, these assumptions assert the existence of a unique equilibrium, and their precise role will be clarified below. Note that we impose no assumption on how the (marginal) revenue depends on $p(i)$ or $T$, but we maintain that marginal costs are increasing in $T$. Intuitively, this means that maintaining a certain market share $p(i)$ at a higher aggregate effort level $T$ requires to bear higher efforts and expenses. ${ }^{6}$

The order assumption (A3) is how we generally introduce heterogeneity to the model. (A3) implies that agents are sorted left-to-right in thus that (marginal) benefits are (weakly) decreasing and (marginal) costs (weakly) increasing in agent index $i$. A common example is that agents are heterogeneous according to their (production) efficiency, such that

$$
\Pi(i)=p(i) V(p(i), T)-c(i) \Phi(p(i), T)
$$

where $c(\cdot)$ is increasing.

\subsubsection{Existence and uniqueness}

Assumptions (A1) and (A2) ascertain the existence of a unique equilibrium.

Theorem 1 (Existence and uniqueness) Any model with payoffs (3) that satisfy assumption 1 has a unique equilibrium $(p(i), T)$. All equilibrium payoffs $\Pi(i)$ are positive, and $p(\cdot)$ is a bounded, decreasing and strictly positive density.

\footnotetext{
${ }^{5}$ This is not problematic because we shall not considering entry or exit decisions of agents.

${ }^{6}$ This will become evident in the context of specific applications.
} 
The proof evolves in two steps, corresponding to the two requirements in the equilibrium definition. The baseline reasoning is illustrated in Figure 1 and goes as follows. (A1) implies that a
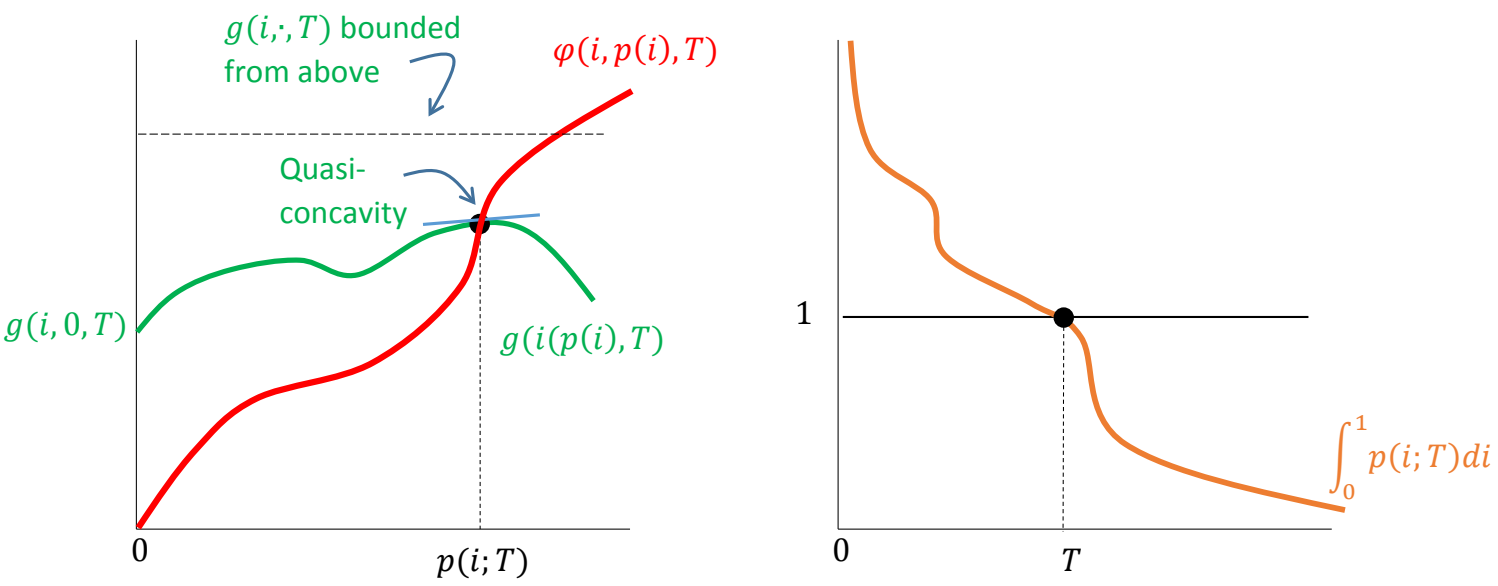

Figure 1: Equilibrium existence and uniqueness

unique optimizer $p(i ; T)>0$ exists for any given $T>0$. This $p(i ; T)$ solves equation (4) for any given $i \in[0,1]$. The existence of such an optimizer follows because a zero market share is not optimal $(g(i, 0, T)>0=\varphi(i, 0, T))$, the gains from increasing one's market share are limited $(g(\cdot, T)$ bounded from above) and marginal costs are strictly increasing in $p(i)$ and unbounded. Uniqueness of this optimizer is implied by strong quasiconcavity. Assumption (A2) then assures that there is a unique $T>0$ such that $\int p(i ; T) d i=1$. To see why, suppose that $g(i, p, T)$ is bounded above and away from 0 for any $p \geq 0$ and any $T>0$, consistent with (but stronger than) assumption (A2). In such a case even the best agent $(i=0)$ seeks to set her market share $p(i ; T)$ arbitrarily small as marginal costs become arbitrarily large $(T \rightarrow \infty)$. Similarly, even the worst agent aims at an arbitrarily large $p(i ; T)$, because marginal costs become arbitrarily small. These two facts imply that $\lim _{T \rightarrow \infty} \int p(i ; T) d i=0$ and $\lim _{T \rightarrow 0} \int p(i ; T) d i=\infty$, and existence of a $T>0$ with $\int p(i, T) d i=1$ follows because $\int p(i ; \cdot) d i$ is continuous. Uniqueness then follows from the last assumption in $(\mathrm{A} 2)$, which assures that $\int p(i ; \cdot)$ is strictly decreasing at $\int p(i, T) d i=1 .{ }^{7}$ Among other, assumption (A3) precludes leap-frogging in equilibrium.

Corollary 1 (Basic equilibrium properties) Consider two agents $i, j \in[0,1]$ with $i<j$. Then in equilibrium $p(i) \geq p(j)$ and $\Pi(i) \geq \Pi(j)$ (no leap-frogging). Moreover, $p(i)>p(j)$ if $g(i, p, T) \geq g(j, p, T)$ and $\varphi(i, p, T) \leq \varphi(j, p, T) \forall p, T>0$, where at least one inequality is strict,

\footnotetext{
${ }^{7}$ This assumption is also essential for the comparative-static results, because it assures, e.g., that $T$ increases if marginal benefits increase exogenously for all agents (Lemma 1).
} 
and $\Pi(i)>\Pi(j)$ if $B(i, p, T) \geq B(j, p, T)$ and $\Phi(i, p, T) \leq \Phi(j, p, T) \forall p, T>0$, where at least one inequality is strict. Further, $p(i)=p(j)$ if both $g(i, p, T)=g(j, p, T)$ and $\varphi(i, p, T)=\varphi(j, p, T)$ $\forall p, T>0$.

The last statement of Corollary 1 implies that if there is a non-trivial interval $\hat{I} \subset[0,1]$ of homogeneous agents, meaning that $B(i, p, T)=B(j, p, T)$ and $\Phi(i, p, T)=\Phi(j, p, T) \forall i, j \in \hat{I}$ and any $p, T>0$, then $p(i)=p(j) \forall i, j \in \hat{I}$. This means that if $[0,1]$ is partitioned by a finite number of non-trivial distinct homogeneous agent intervals $I_{n}$, such that $g(i, p, T) \geq g(j, p, T)$ and $\varphi(i, p, T) \leq \varphi(j, p, T)$, one inequality strict, whenever $j>i, i \in I_{n}$ but $j \notin I_{n}$, then $p(\cdot)$ must be a step-wise decreasing density with finitely many steps, where $p(i)=p(j)$ iff $i, j \in I_{n}$. In such a case we can assume, wlog, that $p(\cdot)$ is right-continuous. In the trivial case, where $n=1$ and thus $I_{1}=[0,1]$, meaning that all agents are homogeneous, the equilibrium is symmetric, i.e. $p(i)=1 \forall i \in[0,1]$. If $g(i, p, T) \geq g(j, p, T)$ and $\varphi(i, p, T) \leq \varphi(j, p, T)$, one inequality strict, for any two $i, j \in[0,1]$ with $i<j$, such that no two agents are identical, then $p(\cdot)$ is a strictly decreasing density.

We summarize this discussion by illustrating the possible structures of $p(\cdot)$ in an example. Suppose that for any $i \in[0,1](2)$ is given by

$$
\Pi(i)=p(i) V(p(i), T)-c(i) C(p(i), T)
$$

where the cost coefficient function $c(\cdot)>0$ either is a finite step-wise increasing function, or a strictly increasing $C^{2}$-function, according to the following definition.

- Class $I$ consists of all increasing, right-continuous step functions for which $\exists i_{0} \in(0,1)$ : $i<i_{0} \leq j \Rightarrow c(i)<c(j)$

- Class II consists of all strictly increasing functions $c \in C^{1}([0,1],[1, \bar{c}])$.

We refer to the final qualification for class I functions as the somewhere strictly increasing (SI) property, which means that $c(\cdot)$ has a step "somewhere in the middle", and is equivalent to the requirement that $c(i)$ is not constant on $(0,1)$. Note that class II functions satisfy SI as well. Class I functions capture the case of finitely many different cost types. That is, the steps partition the population into equivalent cost types, and all members of an equivalence class (i.e., that sit on the same step) are homogeneous to each other and, by Corollary 1, display an 
indistinguishable equilibrium behavior. Intuitively, one could think of every cost type $k$ being "represented" by an agent $i_{k}$, who solves problem (6) for his entire group. The more important interpretation however is that these steps are the continuum analogue to the case of finitely many atomistic agents (see appendix A.1).

The discussion following Corollary 1 shows that if $c(i)$ is class I, a step function capturing agent cost groups $k=1, \ldots, K$ with measures $\gamma_{1}, \ldots, \gamma_{K}>0, \sum_{k} \gamma_{k}=1$, then $p(i)$ will be a corresponding step-wise increasing (density) function, meaning that $p([0,1])=\left\{p\left(i_{1}\right), \ldots, p\left(i_{K}\right)\right\}$ and $\int_{0}^{1} p(i) d i=\sum_{k=1}^{K} \gamma_{k} p\left(i_{k}\right){ }^{8}$ If $c(\cdot)$ is class II, then it follows from Theorem 1, (4) and the Implicit Function Theorem that $p(i)$ must be differentiable density with $p^{\prime}(i)<0$ on $(0,1)$. Summarizing, this means that the respective equilibrium share function $p(\cdot)$ inherits the class membership of $c(\cdot)$.

\section{Heterogeneity: Toolbox and general results}

Throughout section 3.1 we develop some machinery that will help us to analyze how exogenous shocks may affect the equilibrium distribution of market shares. These sections are necessarily of a more technical nature and may be skipped by first or occasional reading. The most important concepts for the later analysis are the definition of rotations (Definition 2), Proposition 2 and Corollary 2.

\subsection{Rotations}

Let $X \subset \mathbb{R}$ be an open parameter interval, $A \equiv[0,1] \times X$, and consider a function $p: A \rightarrow \mathbb{R}_{+}$ with the properties that $\infty>p(0, x) \geq p(1, x)>0$, and $p(\cdot, x)$ is weakly decreasing $\forall x \in X$. In the following, we develop our theory of distributional comparative-statics for the two classes of density functions that naturally emerge with heterogeneous agents.

- Density $p$ belongs to class $\mathbf{I}$ if $p(\cdot, x)$ is a step-wise decreasing, right-continuous density with finitely many steps that has the somewhere strictly decreasing (SSD) property, i.e. $\forall x \in X \exists i_{0} \in(0,1): p(i, x)>p(j, x)$ for $i<i_{0} \leq j$.

\footnotetext{
${ }^{8}$ Hence in case of class I finding the equilibrium generally requires solving a $(K+1)$-system of equations in the unknowns $p\left(i_{1}\right), \ldots, p\left(i_{K}\right)$ and $T$.
} 
- Density $p$ belongs to class II if $p(i, x)$ is $C^{1}$ and $\frac{\partial p(i, x)}{\partial i}<0$ for $i \in(0,1), x \in X$

Class I (II) cost functions as defined in section 2.1.2 yield class I (II) densities. In particular, the SI property of the cost functions implies the SSD property of equilibrium densities. The simplest case of a class I density is the two-types case. If the fraction of "good" (e.g., low-cost) types is $\gamma \in(0,1)$, and we let $i=0$ represent good types and $i=1$ bad types, $p(\cdot)$ has the form

$$
p(\cdot)=\left\{\begin{array}{ll}
p_{0} & i \in[0, \gamma) \\
p_{1} & i \in[\gamma, 1]
\end{array} \quad, p_{1}=\frac{1-\gamma p_{0}}{1-\gamma} \quad, p_{0} \geq p_{1}\right.
$$

How do $p\left(\cdot, x^{\prime}\right)$ and $p(\cdot, x)$ differ in general if $x^{\prime} \neq x$ ? Among the simplest and most interesting movements of $p(\cdot, x)$ as $x$ varies is the idea of rotation.

Definition 2 (Rotations) Let $x \neq x^{\prime} \in X$, and consider the two functions $p\left(\cdot, x^{\prime}\right)$ and $p(\cdot, x)$. We say that $p\left(\cdot, x^{\prime}\right)$ is an outward-rotation $(\mathbf{O R})$ of $p(\cdot, x)$, or $p(\cdot, x)$ is an inward-rotation (IR) of $p\left(\cdot, x^{\prime}\right)$, if $\exists 0<i_{0} \leq i_{1}<1$ such that

$$
\begin{array}{ll}
p\left(i, x^{\prime}\right)>p(i, x) & i \in\left(0, i_{0}\right) \\
p\left(i, x^{\prime}\right)<p(i, x) & i \in\left(i_{1}, 1\right) \\
p\left(i, x^{\prime}\right)=p(i, x) & i \in\left(i_{0}, i_{1}\right)
\end{array}
$$

where the last condition only is required if $i_{0}<i_{1}$. We say that a parameter change $d x>0$ induces a (local) $O R(I R)$ of $p(\cdot, x)$ if $\exists \delta>0$ such that $p\left(\cdot, x^{\prime}\right)$ is $O R(I R)$ of $p(\cdot, x)$ for any $x^{\prime} \in(x, x+\delta)$.

Figure 2 presents some examples of rotations. If $p\left(\cdot, x^{\prime}\right)$ is $\mathrm{OR}(\mathrm{IR})$ of $p(\cdot, x)$, this means that inequality of the market share distribution (or market concentration) has increased (decreased). In particular, in case of an OR those agents who had the largest (smallest) market share under $x$ have an even larger (smaller) market share under $x^{\prime}$, and vice-versa for the IR case.

Given that the decreasing density $p(\cdot, x)$ has the SSD property $\forall x \in X$, the corresponding distribution function $F(\cdot, x)$ is a strictly increasing, continuous and concave function, that is strictly above the $45^{\circ}$-line for $i \in(0,1)$. Moreover, if $p\left(i, x^{\prime}\right)$ is OR of $p(i, x)$, then $F(\cdot, x)$ stochastically dominates $F\left(\cdot, x^{\prime}\right) .^{9}$

\footnotetext{
${ }^{9}$ See Proposition B.1, Online Appendix.
} 

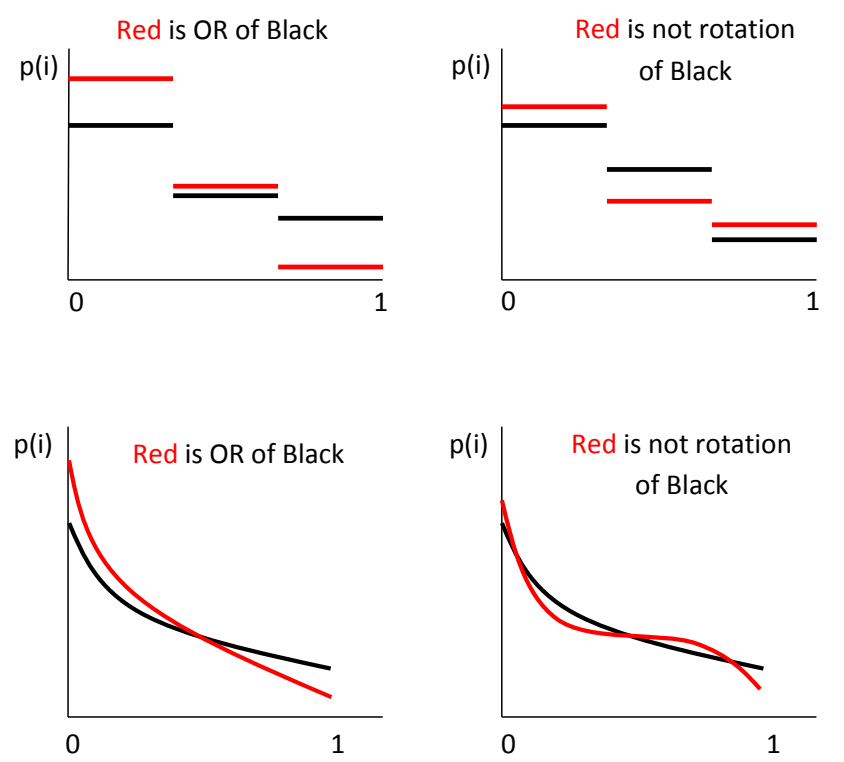

Figure 2: Class I and II rotations

\subsubsection{Detecting rotations}

We now present conditions asserting that $p\left(i, x^{\prime}\right)$ is an OR (or IR) of $p(i, x)$. For a given density on $[0,1]$, let $\left[i_{0}\right]_{x}=\left\{i \in I: p(i, x)=p\left(i_{0}, x\right)\right\}$ the equivalence class of agents with representative $i_{0}$. If $p(\cdot, x)$ is of class $\mathrm{I}$, then there is a finite number of such equivalence classes, and if $p(\cdot, x)$ is of class II, each agent forms her own equivalence class. In any case the union of all equivalence classes form a partition of $I$. Given a density $p(\cdot, x)$, we define the binary relation $\triangleright$ by $j \triangleright i$ if $j>i$ and $j \notin[i]_{x}$. If $p(\cdot, x)$ is of class II, the relations $\triangleright$ and $>$ coincide, and if $p(\cdot, x)$ is of class I, $j \triangleright i$ means that $j$ is of a "worse" (e.g., higher-cost) type than $i{ }^{10}$

If $p\left(\cdot, x^{\prime}\right)-p(\cdot, x)$ is strictly decreasing over its equivalence classes, i.e., if $p\left(\cdot, x^{\prime}\right)-p(\cdot, x)$ is strictly decreasing for any two different agent types, then $p\left(\cdot, x^{\prime}\right)$ is OR of $p(\cdot, x)$ :

Proposition 1 (Difference test) Let $x, x^{\prime} \in X$ and suppose that $\infty>p\left(\cdot, x^{\prime}\right), p(\cdot, x)>0$ are right-continuous, decreasing SSD densities with identical equivalence classes. If

$$
p\left(i, x^{\prime}\right)-p(i, x)>(<) p\left(j, x^{\prime}\right)-p(j, x) \quad \text { whenever } j \triangleright i \in(0,1)
$$

is satisfied, then $p\left(\cdot, x^{\prime}\right)$ is an $O R(I R)$ of $p(\cdot, x)$.

\footnotetext{
${ }^{10}$ Given that in our comparative-static exercises we maintain the initial heterogeneity as, e.g., specified by the cost coefficient function $c(i)$, we do not require to make $\triangleright x$-specific as the equivalence classes typically do not change (but the heights of the steps do).
} 
If $p(\cdot, \cdot)$ is strictly submodular, then (9) is satisfied, but note that if $p(\cdot, x)$ is a step-function, $p(\cdot, \cdot)$ cannot be strictly submodular. ${ }^{11}$ There exists an alternative sufficient condition for the OR-property, which is particularly useful in applications.

Proposition 2 (Ratio test) Suppose that the premise of Proposition 1 is satisfied. If

$$
\frac{p\left(i, x^{\prime}\right)}{p\left(j, x^{\prime}\right)}>(<) \frac{p(i, x)}{p(j, x)} \quad \text { whenever } j \triangleright i \in(0,1)
$$

is satisfied, then $p\left(\cdot, x^{\prime}\right)$ is OR $(I R)$ of $p(\cdot, x)$.

Note that Propositions 1 and 2 encompass both class I and II densities. Comparing conditions (9) and (10) the following observation is useful. ${ }^{12}$ If $u^{\prime}>u>0, v^{\prime}>v>0$ and $\frac{u^{\prime}}{v^{\prime}} \geq \frac{u}{v}>1$ then we have increasing differences, i.e., $u^{\prime}-v^{\prime}>u-v$. Hence, with increasing arguments a (weakly) increasing ratio implies strictly increasing differences. Further, both conditions are equivalent if $p(i, x), p\left(i, x^{\prime}\right)$ are linear in $i$ (which they cannot be if $p(\cdot)$ is of class $\mathrm{I}$ ). In the two-types case (7) properties (9), (10), the OR-property and stochastic dominance of the respective distribution functions are all equivalent:

Proposition 3 (Two-types case) Let $x, x^{\prime} \in X$ and suppose that the densities $p(\cdot, x), p\left(\cdot, x^{\prime}\right)$ are specified by (7) with distribution functions $F(\cdot, x), F\left(\cdot, x^{\prime}\right)$. Then properties (8), (9), (10) and strict stochastic dominance $F\left(i, x^{\prime}\right)>F(i, x)$ are equivalent.

The practical significance of conditions (9) and (10) is that we can derive corresponding differential tests to detect a rotation. This will enable us to use a local criterion to decide about a global property of $p(\cdot)$. We only present the differential version of condition (10) here, because this turns out to be most relevant for our applications; further results are in the Online Appendix (section B.1).

Corollary 2 (Ratio test) Suppose that $p(\cdot)$ is a class I or II density. Further, if $p(\cdot)$ is class I, then $p(i, x)$ is differentiable in $x$, except at step points. Let $x_{0} \in \operatorname{Int}(X)$. If

$$
\frac{\partial}{\partial x}\left(\frac{p(i, x)}{p(j, x)}\right)>0 \quad \forall x \geq x_{0} \text { and any } j \triangleright i \in(0,1)
$$

\footnotetext{
${ }^{11}$ In particular, if $p(\cdot, \cdot)$ is strictly submodular, then $p(\cdot, x)$ must be strictly decreasing $\forall x \in X$. See Online Appendix B.1 for a comment on how the difference and ratio test differ from (log-)supermodularity.

${ }^{12}$ See Online Appendix B.1 for a more general comparison.
} 
whenever the derivative exists, then for any $x>x_{0}(10)$ holds with " $>$ " and hence $p(i, x)$ is OR of $p\left(i, x_{0}\right)$. If the first inequality in (11) is reversed, then, for any $x>x_{0},(10)$ holds with "<", and $p(i, x)$ is IR of $p\left(i, x_{0}\right)$.

By defining

$$
\Delta_{i}(x) \equiv \frac{d p(i)}{p(i, x)}=\frac{\frac{\partial p(i, x)}{\partial x}}{p(i, x)}
$$

condition (11) can be succinctly reformulated as

$$
\Delta_{i}(x)>\Delta_{j}(x) \quad \forall x \geq x_{0} \text { and any } j \triangleright i \in(0,1)
$$

Monotone ratios We say that the function $p(i, x)$ has monotone ratios if for any $x, x^{\prime} \in X$ with $x^{\prime}>x$, condition (10) holds with a fixed inequality for any $j \triangleright i .{ }^{13}$ Monotone ratios yield a particularly pronounced type of rotation, where the effect of $d x$ on $p(i)$ increases monotonically towards the tails of the distribution. Specifically, if $p(i, x)$ has monotone ratios and $p\left(i, x^{\prime}\right)$ is OR of $p(i, x)$, then any agent's relative change in her market share compared to any weaker agent type $j>i$ increases if $x^{\prime} \neq x$. An equivalent interpretation is that the relative change in market shares is strictly increasing in agent type. Hence with an OR (IR) the strongest agents $(i \in i[0])$ gain (lose) most while the weakest agents $(i \in i[1])$ lose (gain) most.

\subsection{Distributional comparative-statics: General Results}

We now use the rotation tools to study how common shocks to the model may affect the distribution of market shares $p(i)$. In section 3.2.4 we show that our setting easily accommodates the case of idiosyncratic shocks. Let $x$ be a (marginal) benefit shifter with $B_{x}(i, p, T, x), g_{x}(i, p, T, x)>$ $0 .{ }^{14}$ Any change in $x$ shifts $g(\cdot)$, potentially by different magnitudes for different agents. The main difficulty in the analysis of how $d x$ affects $p(i)$ is that, in general, $g(\cdot)$ and $\varphi(\cdot)$ both are functions of $p$ and $T$, which in turn are endogenous to the model. A first important stepping stone is to note that the equilibrium aggregate increases in $x$ :

Lemma 1 The equilibrium aggregate $T=T(x)$ verifies $T^{\prime}(x)>0$.

\footnotetext{
${ }^{13}$ If $p(\cdot, x)$ is of class II, then the monotone ratio condition is equivalent to strict log-super(sub)modularity of $p(i, x)$.

${ }^{14}$ For expositional ease we formally consider only benefit shifters. It will become self-evident how to adopt the analysis if $x$ were a cost shifter instead.
} 
$T^{\prime}(x)>0$ holds independent of the equilibrium behavior of $p(i)$. The intuition is quite clear. An exogenous increase in marginal benefits increases efforts of all agents (and thus aggregate effort), since each of them strives for a larger market share. One can note that Lemma 1 also holds if $x$ is an idiosyncratic, rather than a common, shock. In particular, if $M=\{i \in[0,1]$ : $\left.g_{x}(i, p, T, x)>0\right\}$ is a set of positive (Lebesgue) measure and $g_{x}(i, p, T, x)=0 \forall i \in M^{C}$, then $T^{\prime}(x)>0 .{ }^{15}$

\subsubsection{Existence of distributional effects}

We now use (4) and our rotation tools to determine how $p(\cdot, x)$ depends on $x$, always assuming $g(\cdot), \varphi(\cdot)$ to be such that the equilibrium density $p(i)$ is either of class I or II. Let $x_{0} \in X$. Because $p(i, x)$ is implicitly defined by (4) for any $i \in[0,1]$, we have that

$$
g\left(i, p(i), T, x_{0}\right) \varphi(j, p(j), T)=g\left(j, p(j), T, x_{0}\right) \varphi(i, p(i), T) \quad i, j \in[0,1]
$$

or, in short-hand notation, $g(i) \varphi(j)=g(j) \varphi(i)$. In the following we let $i \in[0,1)$ and take any $j \triangleright i$. Total differentiation of (12) with respect to $x$, and rearranging, yields

$$
d p(i)\left(g_{p}(i) \varphi(j)-g(j) \varphi_{p}(i)\right)=d p(j)\left(g_{p}(j) \varphi(i)-g(i) \varphi_{p}(j)\right)+r
$$

where $d p(i)=\frac{\partial p\left(i, x_{0}\right)}{\partial x}$ and

$r=\left(g_{T}(j) T^{\prime}\left(x_{0}\right)+g_{x}(j)\right) \varphi(i)+\varphi_{T}(i) T^{\prime}\left(x_{0}\right) g(j)-\left(g_{T}(i) T^{\prime}\left(x_{0}\right)+g_{x}(i)\right) \varphi(j)-\varphi_{T}(j) T^{\prime}\left(x_{0}\right) g(i)$

Define

$$
\Delta_{i} \equiv \frac{d p(i)}{p\left(i, x_{0}\right)} \quad \varepsilon_{i} \equiv \frac{g_{p}(i) p(i)}{g(i)} \quad \eta_{i} \equiv \frac{\varphi_{p}(i) p(i)}{\varphi(i)}
$$

By strong quasiconcavity (assumption A1): $\eta_{i}>0$ and $\varepsilon_{i}<\eta_{i}$. Using these definitions and (12), (13) can be restated as a surprisingly simple type-recursive relation:

$$
\Delta_{i}=\Delta_{j} k_{i j}+R_{i j}, \quad k_{i j}=\frac{\eta_{j}-\varepsilon_{j}}{\eta_{i}-\varepsilon_{i}}>0
$$

\footnotetext{
${ }^{15}$ This follows readily from the proof of Lemma 1.
} 
where

$$
R_{i j}=\frac{1}{\eta_{i}-\varepsilon_{i}}(A(i)-A(j)), \quad A(s) \equiv\left(\frac{g_{T}(s)}{g(s)}-\frac{\varphi_{T}(s)}{\varphi(s)}\right) T^{\prime}\left(x_{0}\right)+\frac{g_{x}(s)}{g(s)}, \quad s=i, j
$$

Equation (14) decomposes the relation between $\Delta_{i}$ and $\Delta_{j}$ into a direct-aggregative effect ( " $R_{i j}$ "), ${ }^{16}$ and an indirect effect (" $k_{i j}$ "), capturing how marginal benefit and costs depend on $p$. We write $R$ and $k$ instead of $R_{i j}, k_{i j}$ whenever there is no confusion.

Decomposition (14) is key to understanding if and how $d x$ affects the distribution of market shares. Our first main theorem establishes that there are distributional effects of $d x \neq 0$ if and only if $x$ has a non-zero direct-aggregative effect on two different agents $i, j$.

Theorem 2 (Distributional Effects) If $R=0 \forall i, j \in[0,1]$ and any $x \in X$, then $\Delta_{i}=0$ $\forall i \in[0,1]$ and hence $p\left(\cdot, x^{\prime}\right)=p(\cdot, x)$ for any $x, x^{\prime} \in X$. If for a given $x \in X \exists i, j \in[0,1]$ such that $R \neq 0$, then $\exists \delta>0$ such that $p\left(\cdot, x^{\prime}\right) \neq p(\cdot, x)$ for any $x^{\prime} \in(x-\delta, x+\delta)$.

Note that $R=0 \forall i, j \in[0,1]$ iff $A(i)=A(j) \forall i, j \in[0,1]$, i.e., iff $A(\cdot)$ is independent of $i$. As an example, if $g(i)=g(T, x)$ and $\varphi(i)=\varphi(i, p) h(T)$, then $A(s)$ is independent of $s$, and hence $p(i, x)$ is invariant to $x$. More generally, if both $g(i)$ and $\varphi(i)$ are multiplicatively separable $\mathrm{e}^{17}$ in $(i, p)$ and $T$, the bracket in $A(s)$ is independent of $s$. In such a case, $d x \neq 0$ will induce distributional effects if and only if $g(i)$ is not multiplicatively separable in $(i, p)$ and $x$.

\subsubsection{Rotational effects}

We know from Theorem 2 that for $d x$ to induce a distributional effect on $p(\cdot)$, we require that $R \neq 0$ for at least two agents $i \neq j$, but can we say more about how $p(\cdot)$ responds to a (possibly small) change in $x$ ?

Definition 3 For a given $x_{0} \in X$, we say that $R$ is uniformly positive (negative), if $R\left(x_{0}\right)>$ $(<) 0 \forall i, j \in[0,1]$. If $R(x)>(<) 0 \forall i, j \in[0,1]$ holds for any $x \in X$, then $R$ is globally uniformly positive (negative).

\footnotetext{
${ }^{16}$ To understand this terminology, note that $A(i)$ is the total derivative pertaining to (4) with respect to $x$, divided by $g(i)$ and evaluated in equilibrium.

${ }^{17} \mathrm{~A}$ function $\frac{h_{T}(i, p, T)}{h(i, p, T)}$ with $h(\cdot)>0$ is invariant to $i$ if and only if $h(i, p, T)=h_{1}(i, p) h_{2}(T)$. Note that multiplicative separability includes the case where $h(i, p, T)$ does not depend, e.g., on $T$ at all.
} 
The first main result of this section establishes that the direct-aggregative effect $R$, if uniform, is decisive for the resulting distributional pattern of class I densities.

Theorem 3 (Rotational Effects) If $R$ is globally uniformly positive (negative), then for any $x^{\prime}>x_{0}$ we have that $p\left(i, x^{\prime}\right)>p(i, x) \forall i \in[0]$ and $p\left(i, x^{\prime}\right)<p(i, x) \forall i \in[1]$. Moreover, if $p(\cdot, x)$ is of class $I$ and $R$ is uniformly positive (negative) at $x_{0} \in X$, then $d x>0$ induces a local $O R$ (IR) of $p\left(\cdot, x_{0}\right)$.

The first statement in Theorem 3 likewise applies to class I and II densities, and shows how the extreme tails of the distribution evolve for $d x \neq 0$. An important observation then is that the distributional pattern of the two-types case is entirely characterized, at least locally, as a consequence of Theorems 2 and 3 . This follows because in the two-types case $R_{01} \geq(>) 0$ iff $R_{10} \leq(<) 0$, meaning that $R$ is either uniformly positive (negative) or $R=0$. Moreover, it follows from (the proof of) Theorem 3 that if $R$ is globally uniformly positive (or negative) in the three-types case, then any $x>x_{0}$ induces an OR (IR) of $p\left(\cdot, x_{0}\right)$, because the behavior of the "middle group" does not matter.

While the indirect effects (" $k$ " in (14)) are not decisive whether or not $d x$ induces a rotation (given a uniform positive or negative $R$ ), the value of $k$ has implications for the structure of the distributional pattern, as we highlight the next two results. Similar to Definition 3, we say that $k\left(x_{0}\right)$ is uniformly larger (smaller) than one if $k\left(x_{0}\right) \geq(\leq) 1 \forall i, j \in[0,1]$.

Corollary 3 Suppose that $p(\cdot, x)$ is class $I$, and let $R$ be uniformly positive (negative) at $x_{0} \in X$. If $k$ is uniformly larger (smaller) than one, there is $\delta>0$ such that

$$
p\left(i, x^{\prime}\right)>p(i, x) \quad \Rightarrow \quad \frac{p\left(i, x^{\prime}\right)}{p\left(i, x_{0}\right)}>(<) \frac{p\left(j, x^{\prime}\right)}{p\left(j, x_{0}\right)} \quad \forall j \triangleright i
$$

if $x^{\prime} \in\left(x_{0}, x_{0}+\delta\right)$. If $k$ is uniformly smaller (larger) than one, there is $\delta>0$ such that

$$
p\left(i, x^{\prime}\right)<p(i, x) \quad \Rightarrow \quad \frac{p\left(i, x^{\prime}\right)}{p\left(i, x_{0}\right)}>(<) \frac{p\left(j, x^{\prime}\right)}{p\left(j, x_{0}\right)} \quad \forall j \triangleright i
$$

if $x^{\prime} \in\left(x_{0}, x_{0}+\delta\right)$. 
Given that $R$ is uniformly positive, (16) says that among the agents gaining market share, the stronger an agent is (lower index $i$ ), the more the agent gains in relative terms. Similarly, (17) says that among the loosing agents, the weaker the agent is, the more she looses in relative terms.

If $k$ is uniformly equal to one, both statements of Corollary 3 apply, which suggests a complete order across equivalence classes in terms of how the relative market shares change. This applies likewise to class I and class II densities. As Theorem 4, the second main result of this section, shows, this is indeed the case, but there is more. If $k$ is uniformly equal to one, then it is possible to obtain a global characterization of the rotations induced by $d x>0$ for any $x>x_{0}$ by means of the monotone ratio property.

Theorem 4 (Global rotation condition) If $k(x)=1 \forall i, j \in[0,1]$ and any $x \in X$, and $R$ is globally uniformly positive (negative), then $p(i, x)$ has monotone ratios, and $p(i, x)$ is $O R$ (IR) of $p\left(i, x_{0}\right)$ for any $x>x_{0}$.

Theorem 4 applies to class I and II densities likewise. The additional power of the global rotation condition stated in Theorem 4 is that, because of monotone ratios, rotations satisfy a global transitivity condition. If $R$ is globally uniformly positive (negative), then for any $i \in[0,1]$, the relative market share $\frac{p(i, x)}{p(j, x)}, j \triangleright i$, is strictly increasing (decreasing) in $x$, meaning that the market shares will be distributed less and less equally (more and more equally) as $x$ increases.

Inspection of (14) shows that $k(x)=1$ uniformly, and thus $p(i, x)$ has monotone ratios, if both functions $g(\cdot)$ and $\varphi(\cdot)$ have agent-independent $p$-elasticities, i.e., if

$$
g(i, p, T, x)=\hat{g}(i, x, T) p^{\xi(x, T)} \quad \varphi(i, p, T)=\hat{\varphi}(i, T) p^{\zeta(T)},
$$

where the exponents $\xi(x, T), \zeta(T)$ are real-valued functions. ${ }^{18}$ Because the $p$-derivative of a $p$-power function $f(p)=\alpha p^{\gamma}$ is again a $p$-power function, the fact that with agent-independent $p$-elasticities we obtain global rotations with the interesting probabilistic structure of monotone ratios, makes for a strong case to model $B(\cdot)$ and $\Phi(\cdot)$ as power functions of $p$. Power functions indeed constitute a quite flexible function class, particularly because in (18) the exponents need not be constant. As we shall see in section 4, many important examples from several subfields of economics indeed involve power functions and, by Theorem 4, will allow for strong distributional

\footnotetext{
${ }^{18}$ Note that this includes the case, where marginal revenue does not depend on $p$ at all $(\xi(x, T)=0)$.
} 
comparative-static predictions.

\subsubsection{General determinants of the direct-aggregative effect}

Since the direct-aggregative effect is decisive for the distributional pattern of $p(\cdot)$ (Theorems 2 and 3 ), we need to learn more about the main principles that determine $\operatorname{sign}(R)$. For simplicity, we assume that $\varphi(i)=\varphi(i, p) C(T)$ throughout the remainder of this section. Many applications naturally imply such a structure of the cost function (see section 4). If $\varphi(i)=\varphi(i, p) C(T)$, then $\operatorname{sign}(A(i)-A(j))$ depends only on $g(\cdot)$. The next proposition states general principles that determines whether $R$ is (uniformly) positive or negative.

Proposition 4 Suppose that $\varphi(i)=\varphi(i, p) C(T)$, let $g(\cdot)$ be a $C^{2}$-function and define $h(i, p, T, x)=$ $\operatorname{Ln}(g(i, p, T, x))$. If $h_{x}\left(i, p, T, x_{0}\right) \geq h_{x}\left(j, p, T, x_{0}\right), h_{T}\left(i, p, T, x_{0}\right) \geq h_{T}\left(j, p, T, x_{0}\right)$ for all $i, j \in$ $(0,1)$ with $j \triangleright i$, and $h_{T}\left(i, p^{\prime}, T, x_{0}\right) \geq h_{T}\left(i, p, T, x_{0}\right), h_{x}\left(i, p^{\prime}, T, x_{0}\right) \geq h_{x}\left(i, p, T, x_{0}\right)$ for any $i \in(0,1)$ and $p^{\prime}>p$, where at least one of the above inequality is strict, then $R$ is uniformly positive at $x_{0}$. If all inequalities are reversed (and one strict so), then $R$ is uniformly negative at $x_{0}$. Moreover $R$ is globally uniformly positive (negative) if these inequalties hold $\forall x \in X$.

A shock $d x>0$ induces all agents to seek for a larger market share which also increases the aggregate $T$. Incentives to increase market shares are relatively stronger for strong agent types if and only if marginal benefits increase proportionally more for these types, i.e., iff $d g(i) / g(i)>d g(j) / g(j)$ holds for $j \triangleright i$. This is why a uniform change in marginal benefits $(d g(i)=d g(j))$ together with heterogeneity $g(i)>g(j)$ causes an IR $(R<0)$ of $p(i)$, while in case of a proportional change $d g(i) / g(i)=d g(j) / g(j)$ there are no distributional effects. The possibly differential incentive effects of $d x>0$ are either reinforced or weakened by the equilibrium change in aggregate effort. Particularly, if $g_{T}>(<) 0$, then $R>0$ is more likely to result if the increase (decrease) in marginal benefits triggered by $d T>0$ affects the strong agent relatively more (less). In the special case, where marginal benefits are a non-idiosyncratic $p$-power function,

$$
g(i, p, T, x)=u(i) v(T, x) p^{\xi(T, x)},
$$

Proposition 4 implies that $R$ is determined solely by the elasticity function $\xi(T, x)$. Particularly, $R$ is uniformly positive if $\xi_{T}, \xi_{x} \geq(\leq) 0$ with one strict inequality, and $R=0$ uniformly if $\xi$ is constant. 


\subsubsection{Idiosyncratic shocks}

The above type of analysis can be extended to the case of an idiosyncratic (rather than a common) effect of $d x$, which we illustrate in a simple example (see section 4.1.2 for another example). Let $p(\cdot, x)$ be of class I, and let $g(i)=g(i, x)$ and $\varphi(i)=c(i) p(i)^{\eta} h(T)$, such that $k_{i j}=1$ everywhere. Suppose that $d x>0$ only for agents $i \in[m]$, where $m \in(0,1)$. That is, $g_{x}\left(i, x_{0}\right)>0 \forall i \in[m]$ and $g_{x}\left(i, x_{0}\right)=0$ else. ${ }^{19}$ Then $R_{m j}\left(x_{0}\right)>0 \forall j \triangleright m, R_{i m}\left(x_{0}\right)<0 \forall m \triangleright i$, and $R_{i j}\left(x_{0}\right)=0 \forall i, j \notin[m]$. It then follows from (14) that $\Delta_{i}\left(x_{0}\right)>0 \forall i \in[m]$ while $\Delta_{j}\left(x_{0}\right)<0$ $\forall j \notin[m]$. Hence we obtain the intuitive result that type $m$ gains market shares while all other types lose market shares. Moreover, because we must have that $\Delta_{i}=\Delta_{j}$ for $i, j \notin[m]$ the other types lose market share by approximately the same proportion.

\section{Applications}

We give an array of examples, each of which we believe is of self-interest, that use our approach to analyze the distributional comparative statics of the respective model. Clearly, we must restrict ourselves to a subset of possible comparative-statics throughout this exposition; and many of them may be worth exploring further in stand-alone contributions.

\subsection{Monopolistic Competition}

\subsubsection{CES-Demand}

Suppose that there is a continuum $[0,1]$ of consumers, indexed by $\iota$, each endowed with CES utility of the form

$$
U(\iota)=\int_{0}^{1} r_{s} q_{s}(\iota)^{\sigma} d s, \quad \iota \in[0,1]
$$

where $s \in[0,1]$ is a differentiated product supplied by a single firm, also labeled by $s$. Further, $r_{s}>0$ measures the importance of product $s$ to any consumer, ${ }^{20} q^{s}(\iota) \geq 0$ is the respective quantity demanded by $\iota$, and $\sigma \in(0,1)$ is the elasticity of substitution. Each consumer is endowed with disposable income $I(\iota)>0$, and chooses each $q_{s}(\iota), s \in[0,1]$, to maximize (19) subject to $\int P_{s} q_{s}(\iota) d s=I(\iota)$, where $P_{s}>0$ is the price of product $s$. Setting $\eta \equiv \frac{1}{1-\sigma}>1$, and

\footnotetext{
${ }^{19}$ If $[m] \neq[0]$ we additionally assume that $d x>0$ is such that it does not change the order of market shares (i.e. $p\left(\cdot, x^{\prime}\right)$ remains a decreasing density).

${ }^{20}$ For example, $r_{s}$ could be the (relative) quality of variety $s$.
} 
summing up consumer demand $q_{i}(\iota)$ for product $i \in[0,1]$ yields

$$
q_{i}=\frac{I r_{i}^{\eta} P_{i}^{-\eta}}{\int r_{s}^{\eta} P_{s}^{1-\eta} d s}, \quad I \equiv \int I(\iota) d \iota>0
$$

To write firm profits $\Pi(i), i \in[0,1]$, in a way consistent with our framework, let $T \equiv \int r_{s}^{\eta} P_{s}^{1-\eta} d s$, $p(i) \equiv \frac{r_{i}^{\eta} P_{i}^{1-\eta}}{T}$ and for supply costs $\Phi\left(i, q_{i}\right)=c(i) q_{i}^{\gamma_{i}}, c(i)>0, \gamma_{i} \geq 1$. This includes the standard case of constant marginal production costs. Then

$$
q_{i}=\frac{I}{r_{i}^{\frac{\eta}{\eta-1}}} p(i)^{\frac{\eta}{\eta-1}} T^{\frac{1}{\eta-1}}
$$

and

$$
\Pi_{i}=P_{i} q_{i}-c(i) q_{i}^{\gamma_{i}}=I p(i)-I^{\gamma_{i}} \frac{c(i)}{r_{i}^{\frac{\gamma_{i} \eta}{\eta-1}}} p(i)^{\frac{\gamma_{i} \eta}{\eta-1}} T^{\frac{\gamma_{i}}{\eta-1}},
$$

where $p(i)$ is the market share of total income that firm $i$ obtains. From a formal viewpoint, competition in the CES-model is akin to a contest for expenditure shares (see section 4.3). Each firm chooses its market share $p(i)$ (or equivalently its price $P_{i}$ given its demand (20)) to maximize (21), which yields the FOC

$$
\left(\frac{\eta-1}{\eta \gamma_{i}}\right)^{\eta-1} I^{\left(1-\gamma_{i}\right)(\eta-1)}=\frac{c(i)^{\eta-1}}{r_{i}^{\gamma_{i} \eta}} p(i)^{\eta\left(\gamma_{i}-1\right)+1} T^{\gamma_{i}}
$$

We discuss the distributional comparative statics of the CES model by considering separately the case of homogeneous and heterogeneous production elasticities. The results from section 3 allow us to analyze the distributional patterns without the need to explicitly solving the model (which in general is not possible).

Homogeneous production elasticities Suppose that $\gamma_{i}=\gamma \geq 1, \forall i \in[0,1]$. Then (22) implies that $k_{i j}=1 \forall i, j \in[0,1]$. Therefore, by Theorem 4, equilibrium market shares $p(i)$ must have the monotone ratio property whenever the direct-aggregative effect $R$ is either globally uniformly positive or negative. The power-function property of $\varphi(i, p(i), T)$ follows essentially from the constant substitution elasticity $\sigma$. Suppose that $c(i) \leq c(j)$ and $r(i) \geq r(j)$ whenever $i<j$, such that $p(i) \geq p(j)$ in equilibrium. ${ }^{21}$ Moreover, let $c(i)<c(j)$ or $r(i)>r(j)$ for some

\footnotetext{
${ }^{21}$ It is easy to verify that the CES verifies (A1) and (A2). Thus, by Theorem 1, a unique equilibrium exists.
} 
$i, j \in[0,1]$ such that $p(i)$ either is class I or II. Our first result states that equilibrium expenditure shares become more unequal the stronger substitutes the products are or the less elastic the cost function is, while there is no such effect of income, quality or production efficiency.

Proposition 5 The CES-model with homogeneous production elasticities has the following distributional comparative-statics:

\begin{tabular}{|l|c|c|c|c|c|}
\hline & $p(\cdot)$ & $\frac{p(i)}{p(j)}$ & $\frac{\Pi(i)}{\Pi(j)}$ & $\frac{P(i)}{P(j)}$ & $\int \Pi(i) d i$ \\
\hline$d \sigma>0$ & OR & + & + & $\gamma>1: \operatorname{sign}\left(\frac{r(i)}{r(j)}-\frac{c(i)}{c(j)}\right)$ & - \\
\hline$d \gamma>0$ & IR & - & - & $\gamma>1: \operatorname{sign}\left(\frac{r(i)}{r(j)}-\frac{c(i)}{c(j)}\right)$ & + \\
\hline$d I>0$ & 0 & 0 & 0 & 0 & + \\
\hline$d c, d r>0$ & 0 & 0 & 0 & 0 & 0 \\
\hline
\end{tabular}

Table 1: Comparative statics: CES

The last row of Table 1 considers the case of either a common cost (or quality) shock, where $c(i)=c(r(i)=r)$ and $d c>0(d r>0)$.

Equilibrium market shares $p(i)$ are invariant to $d I, d c, d r$, and it follows from the proof of Proposition 5 that $\Pi(i)$ is invariant to $d c, d r$ as well. Stronger substitutes $(d \sigma>0)$ makes competition more intense which, intriguingly, lets already rich firm expand their market size and may marginalize small firms further. An interesting observation pertains to relative prices. In general, $P_{i} \gtreqless P_{j}$ is possible, meaning that relative prices can move quite independently from market shares and relative profits (and vice-versa) in this model. With linear production costs $(\gamma=1)$, we obtain that $\frac{P_{i}}{P_{j}}=\frac{c(i)}{c(j)} \leq 1$, showing that in this case relative prices do not depend on the substitution parameter $\sigma$ at all. Similarly, prices are invariant to the distribution of quality $r(i)$. Moreover, if heterogeneity is entirely driven by quality $(c(i)=c(j))$, then even $P_{i}=P_{j}$ for any quality distribution. This changes with nonlinear production $(\gamma>1)$, because then $P_{i}>P_{j}$ whenever $c(i)=c(j)$ but $r(i)>r(j)$, and the change in relative price given $d \sigma$ depends entirely on the quality-cost ratios.

Heterogeneous production elasticities We now consider the more challenging case, where heterogeneity originates from different cost elasticities $\gamma_{i}$, and discuss the cases of a common 
efficiency $\operatorname{shock}^{22}(d c<0)$ and a positive income shock $(d I>0)$. Contrary to the homogeneous elasticity case, both shocks imply an OR of $p(i)$, and an increase in relative profits and prices. Moreover, $d c<0$ now generates winners $(d \Pi(i)>0)$ and losers $(d \Pi(j)<0)$, and an increase in income yields quantity growth together with a growing inequality of market shares.

For simplicity, we set $r(i)=1$ and $c(i)=c>0 \forall i \in[0,1]$, and assume that $\gamma(\cdot) \geq 1$ is an increasing, non-trivial finite step function. Moreover, we assume parameters such that $q(i) \geq 1$ $\forall i \in[0,1],{ }^{23}$ which by optimality $(22)$ and $\left(20^{\prime}\right)$ then assures that $p(i)>p(j)$ for $j \triangleright i$, such that $p(i)$ is class I. This also implies that $P_{i}<P_{j}$ and $q_{i}>q_{j}$ for any $j \triangleright i$. Because for any given $T>0,(22)$ implies that $\frac{\partial p(i ; T)}{\partial I}, \frac{\partial p(i ; T)}{\partial c}<0, T^{\prime}(I), T^{\prime}(c)<0$ follow. Evaluating (15) for (22) and $x=c$ analogously gives

$$
\operatorname{sign}\left(R_{i j}\right)=\operatorname{sign}(A(i)-A(j))=\operatorname{sign}\left(\left(\gamma_{j}-\gamma_{i}\right) \frac{T^{\prime}(c)}{T}\right), \quad j \triangleright i,
$$

showing that $d c<0$ together with heterogeneous elasticities causes an OR of $p(\cdot)$ by Theorem 3. Using (20') in (22) and rearranging yields

$$
\frac{\eta-1}{\eta}\left(\frac{I}{T}\right)^{\frac{1}{\eta}}=\gamma_{i} c q_{i}^{\left(\gamma_{i}-1\right)+\frac{1}{\eta}} \quad \text { and } \quad \gamma_{i} q_{i}^{\left(\gamma_{i}-1\right)+\frac{1}{\eta}}=\gamma_{j} q_{j}^{\left(\gamma_{j}-1\right)+\frac{1}{\eta}}
$$

Because $d c<0$ triggers an OR and $d T>0$, (20') implies that $d q_{i}>0$ for some $i$ and, by the 2nd equation in (23), hence $d q_{i}>0 \forall i \in[0,1]$. But $d q_{i}>0$ further implies, again by (20'), that $\eta \Delta_{i}+\Delta_{T}>0$, hence also $d(p(i) T)>0$, which by $p(i) T=P_{i}^{1-\eta}$ assures that $d P_{i}<0$. This shows that $d c<0$ increases all quantities supplied and decreases all equilibrium prices, in a way that relative quantities $\frac{q_{i}}{q_{j}}\left(\right.$ use $\left.\left(20^{\prime}\right)\right)$ and relative prices $\frac{P_{j}}{P_{i}}$ must increase. Because $\Pi(i)=p(i) I\left(\frac{\gamma_{i} \eta-\eta+1}{\gamma_{i} \eta}\right)$ it follows that $\frac{\Pi(i)}{\Pi(j)}$ increases, too. Since, by the OR, $p(i)$ increases for $i<i_{0}$ and decreases for $i>i_{0}$, there must be winners and losers, where firm $i$ wins iff it increases its equilibrium market share. This occurs, in contrast to the homogeneous elasticity case, because $d c<0$ allows large firms to decrease their prices by relatively less $\left(P_{i} / P_{j}\right.$ increases), reflecting their relative advantage in the production process, while their quantity $q_{i}$ expands by relatively more. It follows that in such a CES economy, the most dominant firms would also have the strongest incentive to innovate, if this allows to reduce $c$, even if this spills over to

\footnotetext{
${ }^{22}$ An alternative interpretation is that each $q_{i}$ is produced with a single-input technology, where $c$ is the competitive factor cost.

${ }^{23}$ This can be assured, e.g., by assuming that $I$ is large (or $c$ small) enough.
} 
their competitors, and laggards are always hurt by such innovations. The case $d I>0$ is more intricate, and we summarize our main result as a proposition.

Proposition 6 An increase in income $d I>0$ triggers an $O R$ of $p(\cdot)$, increases all quantities $q_{i}$ and also increases relative quantities $\frac{q_{i}}{q_{j}}$, relative profits $\frac{\Pi_{i}}{\Pi_{j}}$, relative market shares $\frac{p(i)}{p(j)}$ and relative prices $\frac{P_{j}}{P_{i}}$ for any $j \triangleright i$.

\subsubsection{Logit-Demand}

In this section we analyze the distributional comparative statics implied by the Logit model with heterogeneous quality. In this model, expected demand from a given consumer is a choice probability system (Anderson et al., 1992). Specifically, we consider the Logit demand-system of a single consumer (or a unit mass of identical consumers) ${ }^{24}$ with linear utility and Logit noise parameter $\lambda>0$, and single-product suppliers. There is no consumer outside option. ${ }^{25}$ Firms $i \in[0,1]$ offer possibly different qualities, parametrized by $a(i) \geq 0$. A firm's market share then is its selling chance

$$
p(i)=\frac{e^{\lambda\left(a(i)-P_{i}\right)}}{\int e^{\lambda\left(a(s)-P_{s}\right)} d s},
$$

where $P_{i}$ is the price of product $i$. Hence increasing the own price reduces $p(i)$, while a higher quality level $a(i)$ increases this probability. Assuming risk neutrality, a constant production cost $c(i)$ of the good, and setting $T \equiv \int e^{\lambda\left(a(s)-P_{s}\right)}$, the (expected) profit of firm $i$ then is

$$
\Pi_{i}=P_{i} p(i)-c(i) p(i)=\left(a(i)-\frac{\operatorname{Ln}(p(i) T)}{\lambda}\right) p(i)-c(i) p(i)
$$

We assume that $a(\cdot)$ is decreasing and $c(\cdot)$ is increasing in a way that $p(i)$ is either of class I or II. Each firm chooses its market share (or equivalently its price subject to expected demand (24)) to maximize its profits. The FOC of this problem $\operatorname{are}^{26}$

$$
a(i)-\frac{\operatorname{Ln}(p(i) T)}{\lambda}-\frac{1}{\lambda}=c(i)
$$

\footnotetext{
${ }^{24}$ If there is a measure $L>0$ of identical consumers, then $p(i) L$ would be the fraction of consumers served by firm $i$. With respect to the distributional outcome, setting $L=1$ is wlog.

${ }^{25}$ In the Online Appendix B.2 we show that the distributional comparative-statics of the Logit model with outside option are similar.

${ }^{26}$ Exponentiating (26), one can easily see that this model verifies (A1) and (A2), and hence a unique equilibrium exists by Theorem 1 .
} 
from which

$$
p(i)=\frac{e^{\lambda(a(i)-c(i))}}{e T}=\frac{e^{\lambda z(i)}}{e T}, \quad z(i) \equiv a(i)-c(i) .
$$

Hence

$$
\frac{p(i)}{p(j)}=e^{\lambda(z(i)-z(j))}
$$

and

$$
\frac{\partial}{\partial \lambda} \frac{p(i)}{p(j)}=e^{\lambda(z(i)-z(j))}(z(i)-z(j))
$$

where the assumptions made on $a(i), c(i)$ assure that $z(i)-z(j)>0$ for any $j \triangleright i$. This shows that the ordered ratio property holds with the Logit, and an increase of $\lambda$ yields an OR of $p(\cdot)$. The parameter $\lambda>0$ controls the noise in the logit. ${ }^{27}$ In the degenerate case where $\lambda=0$, the choice process is purely random in such that neither price nor quality have any influence on choice probabilities, and uniform market shares $(p(i)=1)$ result in any equilibrium. An increase in $\lambda$ means that the price, i.e., the deterministic part of utility, becomes more decisive, which always is associated with an increasing inequality of the market shares. This is a similar finding as with the CES, where an increase in the substitution elasticity (which also makes prices more decisive in a sense) leads to an OR. It follows from (26) that $P_{i}=\frac{1}{\lambda}+c(i)$, showing that prices always decrease in $\lambda$, and

$$
\operatorname{sign}\left(\frac{\partial}{\partial \lambda} \frac{P_{i}}{P_{j}}\right)=\operatorname{sign}(c(i)-c(j)) \leq 0
$$

Because $\Pi(i)=\frac{p(i)}{\lambda}, d \lambda>0$ has the following effects: Relative payoffs increase in $\lambda$, industry profits $\int \Pi(s) d s=\frac{1}{\lambda}$ decrease; hence there must always be losers $(d \Pi(i)<0$ must hold for some firms). The interesting converse is that the weakest firms (in terms of low quality or high costs) have the strongest incentive to increase the "noisiness" in the choice procedure $(d \lambda<0)$, e.g., by resorting to obfuscation tactics (Ellison and Wolitzky, 2012; Hefti, 2016a).

Finally, we prove that $d \lambda>0$ can generate winners, provided that there is sufficient firm heterogeneity. From

$$
\Pi(i)=\frac{1}{\lambda \int e^{\lambda(x(s))} d s}, \quad x(s) \equiv z(s)-z(i) \leq 0,
$$

\footnotetext{
${ }^{27}$ The following is related to the scale-dependence of the logit. If for each $i, j$ we have $v=\frac{\alpha_{i}-c_{i}}{\alpha_{j}-c_{j}}>1$, this means that $d v>0$ has the same effect as $d \lambda>0$.
} 
we obtain that

$$
\operatorname{sign}\left(\frac{\partial \Pi(i)}{\partial \lambda}\right)=\operatorname{sign}\left(-\int e^{\lambda(x(s))} d s-\lambda \int x(s) e^{\lambda(x(s))} d s\right)
$$

With symmetric firms we must have $\frac{\partial \Pi(i)}{\partial \lambda}<0$ as then $x(s)=0$. To see that profits of the best firms can increase in $\lambda$, consider the two-types case with $x(0)=0$ and $x(1)=z(1)-z(0)=C<0$. If $\alpha \in(0,1)$ is the fraction of strong types, then $\partial_{\lambda} \Pi(0)>0$ iff $\alpha<-(1-\alpha)(1+\lambda C) e^{\lambda C}$. We can always find $\alpha \in(0,1)$ small enough, such that this inequality is satisfied, provided that $\lambda C<-1$ (enough heterogeneity). Note that because $\partial_{\lambda} \int \Pi(i) d i<0$, the losses of the poor must always outweigh the gains of the rich.

Import competition Suppose now that the domestic market for the products is composed of home ("H") and foreign ("F") firms, that export their products into home at an import tax of $\tau$. Let $i_{H} \in[0,1]$ and $i_{F} \in[0,1]$ index a home and foreign firm, respectively. Then

$$
p\left(i_{\chi}\right)=\frac{e^{\lambda\left(a\left(i_{\chi}\right)-P_{s}^{\chi}\right)}}{\int e^{\lambda\left(a\left(s_{H}\right)-P_{s}^{H}\right)} d s_{H}+\int e^{\lambda\left(a\left(s_{F}\right)-P_{s}^{F}\right)} d s_{F}}, \quad \chi \in\{H, F\},
$$

where $P_{s}^{H}\left(P_{s}^{F}\right)$ is the price of firm $s_{H}\left(s_{F}\right)$, and $\int p(i) d i=\int p\left(i_{H}\right) d i_{H}+\int p\left(i_{F}\right) d i_{F}=1$. With $T \equiv \int e^{\lambda\left(a\left(s_{H}\right)-P_{s}^{H}\right)} d s_{H}+\int e^{\lambda\left(a\left(s_{F}\right)-P_{s}^{F}\right)} d s_{F}$ we obtain

$$
\begin{aligned}
& \Pi\left(i_{H}\right)=\left(a\left(i_{H}\right)-c\left(i_{H}\right)-\frac{\operatorname{Ln}\left(p\left(i_{H}\right) T\right)}{\lambda}\right) p\left(i_{H}\right), \\
& \Pi\left(i_{F}\right)=\left(a\left(i_{F}\right)-\left(\tau+c\left(i_{F}\right)\right)-\frac{\operatorname{Ln}\left(p\left(i_{F}\right) T\right)}{\lambda}\right) p\left(i_{F}\right),
\end{aligned}
$$

which have the same formal structure as (25). It follows that (26) - (28) logically apply also to this version of the model, where we replace $c(i)$ by $c\left(i_{F}\right)+\tau$ if $i_{F}>1 / 2 .{ }^{28}$ Suppose that $p(i)$ is class I, and consider a small tax increase $d \tau>0$ which, for simplicity, does not change the ranking of $p(i)$. By (28) we see that introducing or changing the tax rate $\tau$ has no effects on the relative market shares $\frac{p\left(i_{H}\right)}{p\left(j_{H}\right)}$ of domestic firms nor on the relative domestic market shares of foreign firms $\frac{p\left(i_{F}\right)}{p\left(j_{F}\right)}$, but the relative market shares $\frac{p\left(i_{H}\right)}{p\left(i_{F}\right)}$ of domestic firms to foreign firms increases in $\tau$ (independent of whether $i_{H} \triangleright i_{F}$ or $i_{F} \triangleright i_{H}$ ). Since by $(27)$ we must have $T^{\prime}(\tau)<0$, it follows again from $(27)$ that $p\left(i_{H}\right)$ increases for any domestic firm and $p\left(i_{F}\right)$ decreases for any

\footnotetext{
${ }^{28}$ Note that the following analysis could also be reformulated as finding the effects of an idiosyncratic change of costs, in the spirit of section 3.2.4, for a subset of firms (no H-F-distinction), where $d \tau$ quantifies a non-common cost innovation or regulation.
} 
foreign firm. It follows that all domestic firms benefit from the import $\operatorname{tax}\left(d \Pi\left(i_{H}\right)>0\right)$, but in a way that leaves relative market shares and relative profits of domestic firms unaltered. This essentially follows from domestic firms expanding their quantities. From (22) one can infer that all domestic prices $P_{i}^{H}$ remain constant, while foreign prices $P_{i}^{F}$ increase isometrically with $\tau$.

Finally, we note that $d \lambda \neq 0$ has similar effects as before. An additional interesting insight here is, that if importers (F-firms) are comparably strong (meaning that $z\left(i_{F}\right)-\tau \gg z\left(i_{H}\right)$ ), such that importing firms gain from $d \lambda>0$, then importers have an incentive to reduce the noisiness of consumer choice, e.g. by educating consumers. The opposite holds if importers are comparably weak; we would then expect importers to blur or complicate consumer perception.

\subsection{Perfect competition and general equilibrium}

We first study competition with price-taking firms facing and exogenous demand, and then show that the distributional methods and results from the previous sections can be fruitfully applied to general equilibrium theory.

\subsubsection{Perfect competition with exogenous demand}

Suppose that price-taking firms face a given inverse demand function $P=P\left(\int q(i) d i, x\right)$ with $P_{1}<0$ and $P_{2}>0$, where $q(i) \geq 0$ is firm $i$ 's quantity supplied, and $x$ is a demand shifter. Further, $\Phi(i, q(i))$ is firm $i$ 's cost function, which is strictly concave in $q(i)$. Defining $T \equiv \int q(i) d i$ and the market share $p(i)=q(i) / T$ yields the profit function $\Pi(i)=P p(i) T-\Phi(i, p(i) T)$, with associated FOC $P(T, x)=\varphi(i, p(i) T)$. Because price-taking behavior implies an identical direct effect of $d x \neq 0$ for all firms, we have

$$
A(i)=-\frac{\varphi_{T}(i)}{\varphi(i)} T^{\prime}=-\frac{\varphi_{q}(i, p(i) T) p(i) T}{\varphi(i, p(i) T)} \frac{T^{\prime}}{T} .
$$

Proposition 7 Equilibrium price $P$, quantities $q(i)$ and profits $\Pi(i)$ are strictly increasing in $x$. If $\Phi(i, q)$ is a power function of $q$ with common and constant exponent $\eta$, then market shares, relative profits and relative quantities all are invariant to $x$, but $\Pi(i)-\Pi(j)$ and $q(i)-q(j), j \triangleright i$, both increase in $x$. If $\Phi(i, q)=q^{\eta(i)}$, where $\eta(i)>1$ is an increasing finite step function and $P(T(x), x)>\eta(1)$, then $d x>0$ induces a local OR of $p(i)$, jointly with an increase in relative profits and relative quantities. 
If $\Phi(i, q)=q^{\eta(i)}$ with heterogeneous exponents, the fact that $k_{i j}=\frac{\eta(j)-1}{\eta(i)-1}>1$ implies, by Corollary 3 , that $\frac{p(i, x)}{p(j, x)}$ increases (locally) in $x$ for any $j \triangleright i$, provided that $p(i, x)$ increases in $x$. The case of iso-elastic (but possibly heterogeneous) costs is very relevant. For example, if the output $q(i)$ is produced with an input vector $x(i)$, acquired on competitive factor markets, according to a production function $f(x(i))$ which has (decreasing) returns to scale, then the corresponding cost function is of the form $\Phi(i, q(i))=q(i)^{\eta(i)} w$. Then different $\eta(i)$ amount to differences in the scale technology, where a lower $\eta(i)<\eta(j)$ means that $i$ produces at higher returns to scale than $j$. Also note that in such a case we could never expect to analytically solve the equilibrium system of equations, even if $P(T, x)$ were linear. Nevertheless, our tools provide us with a clear comparative-static distributional prediction.

We now show that a different class of technology, the family of exponential costs, yields an IR of $p(i)$. Let $\Phi(i, q)=e^{c(i) q}, c(i)<c(j)$, such that $P(T, x)=c(i) e^{c(i) p(i) T}$ and $p(i)>p(j)$, as well as $c(i) p(i)>c(j) p(j)$. Then

$$
A(i)-A(j)=T^{\prime}(c(j) p(j)-c(i) p(i))<0
$$

showing that $d x>0$ triggers an IR of $p(\cdot)$. It is easy to check that if $\Phi(i, q)=c(i) e^{q}$, the same conclusion results.

A second example of costs with which $d x>0$ yields an IR are polynomial costs. Suppose that

$$
\Phi(i, q(i))=c(i) \sum_{s=1}^{m} a_{s} q(i)^{s}, \quad m>1, a_{s} \geq 0
$$

such that costs are scaled polynomials of each other. Note that if $a_{\sigma}>0$ for a single $\sigma>1$ and $a_{s}=0 \forall s \neq \sigma$, then (30) is monomial, and $d x>0$ has no effect on $p(i)$ by Proposition 7 . The following proposition shows that in all other cases, $d x>0$ yields an IR of $p(i)$.

Proposition 8 (Polynomial costs) If $\Phi(i, q(i))$ is given by (30), c(i) is an increasing step function and $a_{s}, a_{s^{\prime}}>0$ for at least two $s \neq s^{\prime}$, then $d x>0$ induces a local IR of $p(i)$.

\subsubsection{Partial equilibrium}

Each consumer $i_{c} \in[0,1]$ has quasilinear utility $U\left(i_{c}\right)=m\left(i_{c}\right)+V\left(i_{c}, q\left(i_{c}\right)^{d}\right)$, where $m\left(i_{c}\right)$ is the quantity of the numeraire ("money"), and $q\left(i_{c}\right)^{d} \geq 0$ is the quantity of the consumption 
good with price $P$. Moreover, $V\left(i_{c}, \cdot\right)$ is a strictly increasing and strictly concave $C^{2}$-function, and $v\left(i_{c}, q\right) \equiv \partial_{q} V\left(i_{c}, q\right)$. To avoid boundary problems, we let $m\left(i_{c}\right) \in \mathbb{R}$. Each consumer is endowed with $\omega_{i_{c}}>0$ of money, where $\omega=\int \omega_{i_{c}} d i_{c}$. Each firm $i \in[0,1]$ produces output $q(i)$ with a technology $q(i)=f(i, z(i))$, where $z(i)$ is the amount of the numeraire used as input, acquired on a competitive factor market, and $f(i, \cdot)$ is a $C^{2}$-function with $f(i, 0)=0$, $f_{q}(i, q)>0$ and $f_{q q}(i, q)<0$ (strict concavity), with $\left(q\right.$-)inverse $f^{-1}(i, f(i, z))=z$. Hence $\Pi(i)=$ $P p(i) T-\Phi(i, p(i) T)$ as in the last section, where $\Phi\left(i, q_{i}\right)=f^{-1}(i, z(i))$ is strictly increasing and strictly convex, and $T=\int q(i) d i$. Each firm maximizes its profits, and each consumer maximizes her utility, subject to $P p^{d}\left(i_{c}\right) T^{d}+m\left(i_{c}\right)=\omega_{i_{c}}$, where $T^{d}=\int q\left(i_{c}\right)^{d} d i_{c}$, and $p^{d}\left(i_{c}\right)=q^{d}\left(i_{c}\right) / T^{d}$ is consumer $i_{c}$ 's share of total market demand. ${ }^{29}$ An equilibrium consists of two densities $p(i), p^{d}(i)>0$ with $\int p(i) d i=\int p^{d}(i) d i=1$ and a quantity $T=T^{d}$ such that the following system of FOC's is satisfied:

$$
\begin{array}{rlrl}
P & =v\left(i_{c}, p^{d}\left(i_{c}\right) T\right) & i_{c} & \in[0,1] \\
P & =\varphi(i, p(i) T) & i \in[0,1]
\end{array}
$$

Because all agents are price-takers, a shock to one market side affects the market shares of the other side only over the indirect-aggregative effect. We will illustrate this by means of a tax example. Suppose that $\tau$ is a quantity tax levied on the supply side. Hence (31) becomes

$$
\begin{aligned}
& P-\tau=\varphi(i, p(i) T) \\
& P=v\left(i_{c}, p^{d}\left(i_{c}\right) T\right)
\end{aligned}
$$

Note that $T^{\prime}(\tau)<0 .{ }^{30}$ An introduction (or increase) of $\tau$ now has the following distributional effects. For firms: Note that $A(i)$ is given by (29), hence the results from last section apply. The tax has differential implications for firm-side market shares only if costs are not a commonelastic power function. If $\varphi(i, q)$ has a $q$-elasticity which is increasing (decreasing) in $i$, then an increase in the tax causes an IR (OR) of $p(i)$, while a decrease of $\tau$ (or the introduction of a subsidy) has opposite effects. For consumers: The tax affects prices and equilibrium quantities.

\footnotetext{
${ }^{29}$ By quasilinearity, we need not worry about the firm ownership, and therefore have not included corporate shares in the budget constraints.

${ }^{30}$ This intuitive result can formally be derived as follows. $T=\int \varphi^{-1}(i, P-\tau) d i \equiv T(P, \tau)$, and $T^{d}=$ $\int v^{-1}\left(i_{c}, P\right) d i_{c} \equiv T^{d}(P)$, where $T_{P}(P, \tau)>0, T_{\tau}(P, \tau)<0$ and $T_{P}^{d}(P)<0$. Because $T=T(P, \tau)=T^{d}(P)=T^{d}$ we have $P^{\prime}(\tau)>0$, and thus $T^{\prime}(\tau)<0$ because $T_{P}^{d}(P)<0$.
} 
We have

$$
A^{d}\left(i_{c}\right)=\frac{T^{\prime}(\tau)}{T}\left(-\frac{v_{q}\left(i_{c}, p\left(i_{c}\right) T\right) p\left(i_{c}\right) T}{v\left(i_{c}, p\left(i_{c}\right) T\right)}\right)
$$

and thus (note that $\eta_{i_{c}}^{d}(\cdot)<0$ by concavity)

$$
R_{i_{c} j_{c}}^{d}=\frac{1}{-\eta_{i_{c}}^{d}}\left(A^{d}\left(j_{c}\right)-A^{d}\left(i_{c}\right)\right)=\frac{1}{\eta_{i_{c}}^{d}}\left(\frac{v_{q}\left(i_{c}, p\left(i_{c}\right) T\right) p\left(i_{c}\right) T}{v\left(i_{c}, p\left(i_{c}\right) T\right)}-\frac{v_{q}\left(j_{c}, p\left(j_{c}\right) T\right) p\left(j_{c}\right) T}{v\left(j_{c}, p\left(j_{c}\right) T\right)}\right) \frac{T^{\prime}(\tau)}{T}
$$

Thus the tax has consequences for $p^{d}\left(i_{c}\right)$ only if $V\left(i_{c}, q\right)$ is not a common-elastic power function. For example, with Log-utility $V\left(i_{c}, q\right)=a_{i_{c}} \operatorname{Ln}(1+q), a_{i_{c}}<a_{j_{c}}$, an increase in tax leads to an IR of $p^{d}(i)$, i.e., $d \tau>0$ reduces consumption inequality of the good among consumers. A subsidy therefore tends to increase consumption inequality. A similar finding holds with an efficiency shock. Suppose that $\varphi(i, q)=c(i) \varphi(q), c(i) \leq c(j)$, and now consider a process innovation leading to a lower cost coefficient $\hat{c}(i)<c(i) \forall i \in[0,1]$. Because $P \int \frac{1}{c(i)} d i=$ $\int \varphi(p(i) T) d i \equiv G(T)$, where $G^{\prime}(T)>0$, we have $T=G^{-1}\left(P \int \frac{1}{c(i)} d i\right)$. Therefore, the aggregate supply increases in the innovation, and we have $\hat{T}>T$ in equilibrium because aggregate demand decreases in $P$. It follows that, with Log-utility, the innovation leads to an OR of $p^{d}(i)$, i.e., an increase in the consumption inequality, similar to a subsidy.

\subsubsection{General equilibrium: two applications}

Decreasing resources Consider a single input-output private ownership economy; firms and consumers are indexed as before. Consumers sell their production resources to firms and acquire the consumption good at a price $P$ with their income. All markets are competitive. Let $\omega\left(i_{c}\right)>0$ denote the resource endowment of consumer $i_{c}$, and $S\left(i_{c}\right)=s\left(i_{c}\right) \Pi$ is consumer $i_{c}$ 's share of aggregate profits $\Pi=\int \Pi(j) d j$. Further $\omega=\int \omega\left(i_{c}\right) d i_{c}$ and $\int s\left(i_{c}\right) d i_{c}=1$, where both $\omega(\cdot)$ and $s(\cdot)$ are (weakly) decreasing functions. This will allow us to distinguish between income inequality caused by differences in resources or in capital ownership. Each consumer $i_{c}$ expends his entire income on consumption, which yields a demand $\left(q\left(i_{c}\right)=p^{d}\left(i_{c}\right) T^{d}\right)$

$$
p^{d}\left(i_{c}\right) T^{d}=\frac{\omega\left(i_{c}\right)+S\left(i_{c}\right)}{P}
$$

Aggregate demand therefore is $T^{d}=(\omega+\Pi) / P$. The production process of each firm is described by a cost function as in section 4.2.2. Because firms behave as price-takers, firm FOC 
are given by (31) (2nd equation), and $A(i)$ by (29). The difference to the partial equilibrium model is that now demand and the (relative) consumption price depend on capital income (profit), which in turn depends on demand. In the following we consider the comparative statics if $d \omega\left(i_{c}\right)=d \omega<0 \forall i_{c} \in[0,1]$, i.e., there is a uniform contraction of the available resources. We assume cost functions and $\omega(\cdot), s(\cdot)$ to be such that $p(\cdot), p^{d}(\cdot)$ are of class I.

Proposition 9 (Firms) In equilibrium: $T^{\prime}(\omega), P^{\prime}(\omega)>0$ and $\partial_{\omega} \Pi(i), \partial_{\omega} q(i)>0$. If the $q$ elasticity of $\varphi(i, q)$ is strictly increasing (decreasing) over firm equivalence classes, $d \omega<0$ induces a local IR (OR) of $p(i)$. If $\Phi(i, q(i))=c(i) q(i)^{\eta}$, then $p(i)$ is invariant to $\omega$.

While all firms supply less to the market as the scarcity of the resource increases, it depends in a clear way on the production possibilities whether market concentration increases or decreases. Additionally, it can be shown that if production functions are heterogeneous power functions (such that $\eta(i)$ is increasing), the resulting IR caused by $d \omega<0$ is passed on to the shares of the firms in the factor market. Specifically, $d \omega<0$ induces an IR of the firm's relative shares in the factor market.

The next proposition shows how consumption inequality depends on $\omega$. We consider the two extreme regimes, where consumption inequality originates from i) the resource endowment allocation or ii) from the share allocation.

Proposition 10 (Consumption) (i) If $s\left(i_{c}\right)=1 \forall i_{c}$, then $d w<0$ induces a global OR of $p^{d}(\cdot)$ with monotone ratios, and relative consumption $q^{d}\left(i_{c}\right) / q^{d}\left(j_{c}\right)$ increases. (ii) If $\omega\left(i_{c}\right)=\omega$ $\forall i_{c}$, then $d w<0$ induces a global OR (IR) of $p^{d}(\cdot)$ with monotone ratios, if and only if $\Pi(\omega)$ is strictly concave (convex). Moreover, $q^{d}\left(i_{c}\right) / q^{d}\left(j_{c}\right)$ increases (decreases) if and only if $d \omega<0$ leads to an $O R(I R)$ of $p^{d}(\cdot)$.

If income inequality originates from the share distribution and production is best described by common-elasticity power functions, consumption shares, relative consumption, firm market shares and relative profits all become invariant to $\omega$.

Corollary 4 If $\omega\left(i_{c}\right)=\omega \forall i_{c}$ and $\Phi(i, q(i))=c(i) q(i)^{\eta}$, then $p^{d}(\cdot)$ and $q^{d}\left(i_{c}\right) / q^{d}\left(j_{c}\right)$ are invariant to $\omega$, but $d q\left(i_{c}\right)<d q\left(j_{c}\right)<0$, i.e., absolute consumption inequality decreases.

Note that all propositions apply analogously (with "reversed signs") in case of a resource expansion $d \omega>0$. 
Labor-consumption decisions Consider a perfectly competitive private ownership economy, where a consumption output is produced by firms using only labor as input. Each consumer $i_{c} \in[0,1]$ owns a unit of perfectly divisible labor and decides between consumption $q\left(i_{c}\right) \geq 0$ and leisure $f\left(i_{c}\right) \in[0,1]$ according to utility $u\left(i_{c}\right)=x\left(i_{c}\right)^{\alpha} f\left(i_{c}\right)^{1-\alpha}$. The parameter $\alpha \in(0,1)$ measures how important consumption is relative to leisure. The consumer budget constraint is $q\left(i_{c}\right)=\left(1-f\left(i_{c}\right)\right) w+S\left(i_{c}\right)$, where $w$ is the real wage and $S\left(i_{c}\right)=s\left(i_{c}\right) \Pi \geq 0$ is capital income of corporate shares. As before $s(\cdot) \geq 0$ is decreasing with $\int s\left(i_{c}\right) d i_{c}=1$, and $\Pi=\int \Pi(i) d i$ are aggregate profits. It follows that optimal consumption and leisure choices are given by $q\left(i_{c}\right)=$ $\alpha\left(w+S\left(i_{c}\right)\right)$ and $f\left(i_{c}\right)=\frac{\alpha}{1-\alpha} \frac{q\left(i_{c}\right)}{w}$, respectively. ${ }^{31}$ Let $p^{d}\left(i_{c}\right)=q\left(i_{c}\right) / T^{d}, T^{d}=\int q\left(i_{c}\right) d i_{c}$ deonte $i_{c}$ 's share of total consumption. Each firm $i \in[0,1]$ produces $y(i) \geq 0$ units of the good using only labor as input according to a strictly concave technology, yielding a corresponding strictly convex cost function $\hat{\Phi}(i, q(i))=w \Phi(i, q(i))$ with $\Phi(i, 0)=0$. We suppose that $\Phi(\cdot, q)$ is such that $p(\cdot)$ is of class I (while $p^{d}(\cdot)$ could be of class I or II). The profit is $\Pi(i)=p(i) T-w \Phi(i, p(i) T)$, with FOC $1=w \varphi(i, p(i) T)$. Because the wage is exogenous to each firm, $A(i)$ must be of the type (29). Consequently, the type of rotation of $p(i)$ is determined entirely by the indirect-aggregative effect of a common shock, and $R_{i j}>(<) 0$ if $d T>0$ and the $q$-elasticity of $\varphi(i, q)$ is strictly increasing (decreasing) in $q$. Hence the distributional comparative-statics of firm-side variables obey essentially the same laws as with an exogenous demand (section 4.2.1).

We now seek to analyze how the distribution of consumption and leisure across consumers depends on i) the (common) state of technology in the economy and ii) the importance of leisure as parametrized by $\alpha$. To this end, we begin by setting $\Phi(i, q(i))=c(i) q(i)^{\eta}, \eta>1$, $c(i)>0$, where $c(\cdot)$ is increasing. A common positive technology shock means $d c(i)<0 \forall i$, i.e., production efficiency increases for all firms. ${ }^{32}$ With this technology $R_{i j}=0$, showing that $p(\cdot)$ and relative profits do not depend on the state of technology or $\alpha$. We summarize the main distributional results on the consumer side in the following proposition, where always $j \triangleright i$ and $j_{c} \triangleright i_{c}$.

Proposition 11 (i) A common increase in efficiency $(d c(i)<0)$ increases all equilibrium consumption levels $q\left(i_{c}\right)$, the wage and all profits $\Pi(i)$, while $p^{d}\left(i_{c}\right), p(i), \frac{q\left(i_{c}\right)}{q\left(j_{c}\right)}, f\left(i_{c}\right)$ (and labor

\footnotetext{
${ }^{31}$ This interior solution requires that $S\left(i_{c}\right) \leq \frac{\alpha}{1-\alpha} w$ which, for simplicity, we shall assume to hold. Corresponding precise conditions can be derived, e.g., in the context of our parametric example. Moreover, the main results carry through if the boundary condition $f\left(i_{c}\right) \leq 1$ becomes binding (and thus $q\left(i_{c}\right)=S\left(i_{c}\right)$ ) for some consumers.

${ }^{32}$ More precisely, we mean by $d c(i)<0$ a downward shift of the function $c(\cdot)$ in the sense that $c(i)=\hat{c}(i)+\varepsilon$ and $d \varepsilon<0$.
} 
supply) and relative profits all are invariant to the state of technology, but $d q\left(i_{c}\right)>d q\left(j_{c}\right)$ and $d \Pi(i)>d \Pi(j)$, showing that absolute consumption and profit inequality increases in efficiency. (ii) $d \alpha>0$ induces an OR of $p^{d}\left(i_{c}\right)$, with monotone ratios, and increases $\frac{q\left(i_{c}\right)}{q\left(j_{c}\right)}$ and $\frac{f\left(i_{c}\right)}{f\left(j_{c}\right)}$ as well as all profits, aggregate consumption and aggregate labor supply, while wages fall. Firm market shares $p(i)$ and relative profits are invariant to $\alpha$. Finally, $d \Pi(i)>d \Pi(j)$, and $d q\left(i_{c}\right)>d q\left(j_{c}\right)$ whenever $d q\left(i_{c}\right)>0$.

The intuition in case of an efficiency increase is that this allows firms to produce at lower costs (equivalently: more from a given input), yielding higher supply, higher profits, higher real wages and more consumption. As profits and wages increase proportionally, the incentives to work more and benefit from the higher wage or to rather enjoy more leisure and finance consumption from the higher capital income counterbalance each other, leading to a constant labor supply and constant consumption shares. Because consumption levels increase, $d q\left(i_{c}\right)>d q\left(j_{c}\right)$ follows naturally.

The intuition in case of $d \alpha>0$ is that if consumption is more important, consumers supply more labor to afford more consumption, which reduces real wages and increases profits, which benefits capital owners and therefore increases consumption inequality. Additionally, one can show that because real wages plunge the poorest may even end up with a lower consumption level despite that $d \alpha>0$ increases the propensity to consume more. ${ }^{33}$

We now show that the result on $d \alpha$ generalizes beyond the case where costs are commonelasticity functions. Suppose that $\Phi(i, q(i))$ is not restricted beyond the assumption stated in the beginning of this paragraph, and consider $d \alpha>0$. Then the results on $p^{d}\left(i_{c}\right), q\left(i_{c}\right) / q\left(j_{c}\right)$, $f\left(i_{c}\right) / f\left(j_{c}\right), w, T$ and aggregate labor supply in Proposition 11 remain valid provided that $T \leq w \cdot{ }^{34}$ To see this, write firm FOC as $p(i) T=\varphi^{-1}(i, 1 / w)$. Integration yields

$$
T=\int \varphi^{-1}(i, 1 / w) d i \equiv H(w)
$$

with $H^{\prime}(w)<0$. Setting $T^{d}=T$ implicitly defines $w(\alpha)$ by

$$
H(w(\alpha))=\alpha(w(\alpha)+\Pi(w(\alpha)))
$$

\footnotetext{
${ }^{33}$ If $\Phi(i, q(i))=c(i) q(i)^{\eta}$ and a positive mass of consumers holds no shares at all $(s(i)=0)$ this is the case iff $\alpha(\eta-1)>0$

${ }^{34}$ If $\Phi(i, q(i))=c(i) q(i)^{\eta}$ it can be shown that indeed $T \leq w$ must hold in equilibrium.
} 
By the Envelope Theorem $-\partial_{w} \Pi(i)=\Phi(i, q(i))<q(i) \varphi(i, q(i))=q(i) / w$, where the inequality follows from the strict concavity of $\Phi(i, \cdot)$ and $\Phi(i, 0)=0$, and the equality from firm FOC. Integration yields $-\Pi<T / w$. By (33) we have

$$
w^{\prime}(\alpha)=\frac{w+\Pi}{H^{\prime}(w)-\alpha\left(1+\Pi^{\prime}(w)\right)} .
$$

The denominator is negative if $T \leq w$, because then $1+\Pi^{\prime}(w) \geq 0$, which assures that $w^{\prime}(\alpha)<0$. Then $T^{\prime}(\alpha)>0$, and the remaining claims follow from the proof of Proposition 11.

\subsection{Contests}

Many real-world competitions can best be described as contests for scarce goods. Therefore, we now study how the reward scheme in (imperfectly discriminatory) contests affects the resulting distribution of success chances and related measures.

Consider a unit mass of agents competing to obtain a prize. Each agent can invest effort to win the prize. The prize could be, e.g., obtaining a research grant, winning a political election, or winning a sport championship. In some cases we would expect the value of the prize to depend on the winning or aggregate effort, too. Individual efforts levels and winning chances are positively correlated but, other than in auctions, the highest effort level needs not win the contest with certainty. Such scenarios have been typically analyzed in the literature using a model of (imperfectly discriminatory) contests (Konrad, 2009), with a (general) payoff function

$$
\Pi(i)=\pi(t(i), T) V(t(i), T, x)-\Phi(i, t(i)),
$$

where $\pi(t(i), T), T=\int t(i) d i$, is a Contest Success Function (CSF), and $V(t(i), T, x)$ is the prize function. The CSF captures how individual efforts to seize the prize translate into winning odds, where we always assume that $\partial_{t} \pi(t, T)>0$ on the relevant range. A natural benchmark assumption is that $\pi(\cdot)$ is zero-homogeneous in $(t, T)$, i.e., doubling all effort level leaves individual success chances unchanged. Then, for $T>0$, we let $\pi(t, T)=\hat{\pi}(t / T)$ wlog. Regarding the prize function, we assume that $V(\cdot)>0$ is a $C^{2}$ function. The parameter $x$ is an exogenous prize shifter with $V_{x}>0$ and $V_{x x} \geq 0$. Note that this includes the standard case where $V>0$ is a fixed prize value. We take all functions involved in (34) to be such that Assumption 1 is 
satisfied. A standard example is the Tullock fixed-prize contest with linear effort costs

$$
\Pi(i)=\frac{\hat{t}(i)^{1 / \eta}}{\int \hat{t}(s)^{1 / \eta} d s} V-c(i) \hat{t}(i)
$$

where $\eta \in(0,1)$ quantifies the degree of noise in the Tullock CSF (higher $\eta$ means more randomness in the CSF). Using the monotone transformation $t(i) \equiv \hat{t}(i)^{1 / \eta}$, we obtain the equivalent representation of a linear Tullock CSF with iso-elastic costs

$$
\Pi(i)=\frac{t(i)}{T} V-c(i) t(i)^{\eta}
$$

The distributional comparative-statics of the contest model (34) can be analyzed by the framework of this article, using the transformation $p(i)=\hat{\pi}(t(i) / T)$. Then

$$
\Pi(i)=p(i) V\left(\hat{\pi}^{-1}(p(i)) T, T, x\right)-\Phi\left(i, \hat{\pi}^{-1}(p(i)) T\right)
$$

There are two conceivable interpretations of $p(i)$. First, $p(i)$ is agent $i$ 's probability to seize a single prize worth $V(\cdot)$. Second, $p(i)$ is the market share of agent $i$ and $V(\cdot)$ is the value of the market share to the agent. The two models are formally equivalent, while the notion of a "winner" makes sense in the first interpretation.

Our first result shows that a prize shift induces distributional effects only if $V(\cdot)$ depends on $i$ (through $p(i))$ or $\Phi(i, t(i))$ is not a common-elastic power function.

Proposition 12 Let $\pi(t(i), T)$ be a zero-homogeneous CSF. If the prize function is $V(T, x)$ the distributional patterns of $p(i)$ are determined by the t-elasticity of marginal costs alone. Specifically, if $\Phi(i, t)=c(i) t^{\eta}$, then $p(i), \Pi(i) / \Pi(j)$ as well as $t(i) / t(j)$ are invariant to $x$.

As with quantity competition, if the q-elasticity of marginal costs increases (decreases) over agent equivalence classes, $d x>0$ induces an OR (IR) of $p(i)$. Because the Tullock fixed-prize contest (35) is a special case of Proposition 12, an exogenous change of the prize $d V$ has no distributional effects other than $d t(i)>d t(j)>0$ as well as $d \Pi(i)>d \Pi(j)>0$. The derivation of (34') shows that $\eta$ parametrizes the noise of the CSF in a Tullock contest with an agent-independent prize, and an increase in noise always induces an IR of $p(i)$.

Corollary 5 In the Tullock contest with $\Pi(i)=p(i) V(T)-c(i) p(i)^{\eta} T^{\eta}$, where $c(\cdot)$ is such that 
$p(\cdot)$ is of class I or II, $d \eta>0$ induces a global IR of $p(i)$, with monotone ratios, and $\Pi(i) / \Pi(j)$ as well as $t(i) / t(j)$ both increase in $\eta$.

Hence an increase in randomness of the contest tends to benefit weak contestants more, similar to the finding with Logit demand in section 4.1.2.

In some circumstances the market value $V(\cdot)$ may be thought of depending positively or negatively on the (winning) agent's effort level. For example, the intensity of political lobbying (Konrad, 2009), litigation expenditures (Posner, 1992), salary negotiations (Amegashie, 1999) or money invested to obtain a monopoly franchise (Chung, 1996) can influence the final conditions of the winning agent. Similarly, $V(\cdot)$ can express the market value of consumer attention to a firm (Hefti, 2016a). To unshroud the effects of the $t(i)$-sensitivity of $V(\cdot)$, we let $V(i)=\alpha t(i)+\beta$. In the advertising example, $\alpha \neq 0$ means that advertising intensity $t(i)$ determines market share, e.g., the fraction of consumers paying attention to $i$, but $t(i)$ further affects the willingness to pay of attentive consumers, e.g., by strengthening the attachment or joy experienced by consuming a brand $(\alpha>0),{ }^{35}$ or by increasing the nuisance or intrusion felt by ad exposure $(\alpha<0)$ (Johnson, 2013; Hefti and Liu, 2016).

Proposition 13 Suppose that $V(t(i))=\alpha t(i)+\beta$, where $\beta>0$ and $\alpha \gtreqless 0$. Let $\pi(t(i), T)$ be a zero-homogeneous CSF, and $\Phi(i, t)=c(i) t^{\eta}, \eta>1$, where $c(\cdot)$ is such that $p(\cdot)$ is class $I$. Let $z(p) \equiv \hat{\pi}^{-1}(p)$. Then $d \alpha>0$ induces a local OR (IR) if $\forall p>0: \frac{z^{\prime \prime}(p) p}{z^{\prime}(p)}>(<)-2$. Further, $d \beta>0$ induces a local $O R$ if either $\alpha>0$ and $\frac{z^{\prime \prime}(p) p}{z^{\prime}(p)}<-2$ or if $\alpha<0$ and $\frac{z^{\prime \prime}(p) p}{z^{\prime}(p)}>-2$.

A corollary is that in the Tullock contest $\left(z^{\prime \prime}(p)=0\right), d \alpha>0$ induces an OR with monotone ratios (and an increase in relative efforts and payoffs), while $d \beta>0$ leads to an OR if $\alpha<0$. Because $T^{\prime}(\alpha)>0$, Proposition 13 reveals an interesting efficiency-equity trade-off. For example, if $\alpha$ is a design variable, say in a sport tournament, and the CSF is best described by the Tullock formula, then increasing effort sensitivity of the prize function induces more unequal winning chances (the same teams tend to win all the time), but also increases aggregate effort. Conversely, if the goal is to make the competition less predictable (IR of $p(\cdot)),{ }^{36}$ then a reduction of total effort is unaviodable.

In terms of real-world prediction, in the advertising example we expect to observe more market

\footnotetext{
${ }^{35}$ In such a case, advertising has a persuasive or complementary nature (Bagwell, 2007).

${ }^{36}$ In sports economics, the idea of increasing the competitive balance between two agents, i.e. reducing $p(i) / p(j)$, is a very important concern of optimal tournament designer (Szymanski, 2003).
} 
concentration at higher levels of advertising affinity $(\alpha>0)$ and less market concentration with annoying advertising $(\alpha<0)$ provided that $\hat{\pi}^{-1}(p)$ is not too concave (otherwise the reverse prediction applies). The fact that the curvature of $\hat{\pi}^{-1}(p)$ plays a role is quite intuitive as we exemplify in case of $\alpha>0$. An increase in $\alpha$ increases marginal revenues of advertisers, but more so for high intensity advertisers $\left(t(i)\right.$ high). If $\hat{\pi}^{-1}(p)$ is convex, then marginally increasing the market share is easier the higher the current level of market share is. Hence $d \alpha$ has a stronger incentive effect to increase the market share (advertising) for already strong advertisers, leading to an OR of $p(\cdot)$.

Two-prize contests As a final variation we show that introducing (or increasing) a second prize in a contest tends to make the chances to win the first prize more equal. Contests with multiple prizes have received attention in the contest architecture literature, but typically only the case of symmetric agents is studied. ${ }^{37}$ Consider a contest with two fixed prizes $V_{1}>0$ and $V_{1} \geq V_{2} \geq 0$. Suppose that there is a number $n$ of atomistic agents. ${ }^{38}$ Expected profit of $i$, given an effort profile $(t(1), \ldots, t(n))$, is

$$
\Pi(i)=\frac{t(i)}{T} V_{1}+\frac{t(i)}{T} \sum_{j \neq i} \frac{t(j)}{T-t(j)} V_{2}-\frac{1}{\eta} c(i) t(i)^{\eta}
$$

Then $p(i)=t(i) / T$ is the probability to win the first prize. It can be verified by our standard approach that the overall prize composition $\left(V_{1}, V_{2}\right)$ has no distributional effects on $p(i)$, provided that there are only two cost types. It turns out that this is not valid in general. Already with three cost types it is possible to construct examples where $p(i)$ varies with the prize composition.

The general difficulty with (36) is that if there are more than two cost types, (36) is a function not only of $t(i)$, but also of all $t(j)$ with $j \neq i$. By imposing the simplifying assumption of sampling with (instead of without) replacement for the second prize, the two-prize contest fits into the present framework, and (36) becomes

$$
\Pi(i)=\frac{t(i)}{T} V_{1}+\frac{T-t(i)}{T} \frac{t(i)}{T} V_{2}-\frac{1}{\eta} c(i) t(i)^{\eta},
$$

\footnotetext{
${ }^{37}$ For example Clark and Riis (1998) consider the case of a multi-prize contest with symmetric contestants. Their main interest is aggregate effort, and they find that highest aggregate effort requires to award only one prize.

${ }^{38}$ It is easier to set up the problem with discrete agents. The respective optimality conditions can then be easily given a "continuum interpretation" such that our distributional tools can be applied.
} 
where $\frac{T-t(i)}{T} \frac{t(i)}{T}$ is the chance of $i$ to win the second prize. ${ }^{39}$ Replacing $t(i)$ by $p(i) T$ in $(37)$ and differentiating with respect to $p(i)$ yields the FOC

$$
V_{1}+(1-2 p(i)) V_{2}=c(i) p(i)^{\eta-1} T^{\eta}
$$

It turns out that with (38) we can obtain a characterization of how changes in the prize scheme $\left(V_{1}, V_{2}\right)$ affect the distribution of the first-prize winning chances.

Proposition 14 If $\frac{d V_{1}}{V_{1}}>(<) \frac{d V_{2}}{V_{2}}$ a local OR (IR) results. If $\frac{d V_{1}}{V_{1}}=\frac{d V_{2}}{V_{2}}, p(i)$ remains constant. Proposition 14 suggests that an unilateral change of a single prize in presence of another prize always in general has distributional consequences. An unilateral increase of the first (second) prize implies more inequality (equality) in the first-prize winning chances $p(i)$, and if the overall prize money $V=V_{1}+V_{2}$ is increased, both prizes should be increased proportionally if $p(i)$ is to be kept constant. Proposition 14 further provides insights on how changing the composition of a given overall prize sum $V=V_{1}+V_{2}>0, V_{1} \geq V_{2} \geq 0$, affects $p(i)$ and thus relative efforts $t(i) / t(j)$. The fact that $d V_{1}<0$ and $d V_{2}>0$ both imply an IR suggests that the case of an even prize split $V_{1}=V_{2}=V / 2$ generates most equality, while $V_{1}=V$ (a single-prize contest) induces inequality. Moreover, there is an interesting effort-equality trade-off because at the same time aggregate effort $T\left(V_{1}, V_{2}\right)$ decreases if $V_{2}=V-V_{1}$ increases. ${ }^{40}$

Analyzing how the distribution of equilibrium payoffs depends on the prizes turns out to be tricky in general. However, in the special case where $\eta=2$ it is possible to show that $\frac{\Pi(i)}{\Pi(j)}=\frac{p(i)}{p(j)}$. It follows that relative payoffs increase (decrease) whenever $\Delta_{i}>\Delta_{j}$. Since $d V_{1}>0$ causes an OR, it follows that $\frac{\Pi(0)}{\Pi(1)}$ increases, while this ratio decreases if $d V_{2}>0$. Hence moving from a single prize two two equal prizes tends to squish payoffs together. Finally, one could also ask how the overall chances to win any prize depend on $\left(V_{1}, V_{2}\right)$. The chance of agent $i$ to win a prize is $s(i) \equiv p(i)+(1-p(i)) p(i)$, and thus $d s(i)=2 d p(i)(1-2 p(i))$. Assuming that there is not so much asymmetry that $p(i) \geq 1 / 2, s(i)$ behaves like $p(i)$ since $\operatorname{sign} d s(i)=\operatorname{sign} d p(i)$.

\footnotetext{
${ }^{39}$ We would expect these sampling chances to be close to those in (36) for a large number of agents. For example, if $t(j)=t>0 \forall j$ then the absolute difference between the two is of order $1 / n^{2}$.

${ }^{40}$ To see that $T\left(V_{1}, V-V_{1}\right)$ decreases in $V_{1}$, use $V_{2}=V-V_{1}$ in (38) and note that $\frac{\partial}{\partial V_{1}} p(i)>0$.
} 


\subsection{Relation to Nash equilibrium}

In the previous sections we analyzed a contest model under the assumption that individual agents take the aggregate $T=\int t(i) d i$ as given when choosing their effort (equivalently: their equilibrium market share), while $T$ is still endogenous to the model. One justification is that contestants have a good intuition about the average (or aggregate) effort imputed in equilibrium while they do not know the cost functions (the strategies) of the competitors. ${ }^{41}$ We now demonstrate, by means of the contest example, that our distributional tools can be successfully applied to the concept of Nash equilibrium. ${ }^{42}$

Consider first a fixed-prize contest with $n$ atomistic agents and payoffs

$$
\Pi(i)=\pi\left(\frac{t(i)}{\sum t(s)}\right) V-\Phi(i, t(i))
$$

Define the market share $p(i)=\pi(t(i) / T)$ as before. The only difference is that each agent $i$ now takes into account its own effect on the aggregate. Let $T_{i} \equiv \sum_{s \neq i} t(s)$ and $z(p(i)) \equiv \pi^{-1}(p(i))$. Because $T=T_{i}+t(i)$ and $t(i)=z(p(i)) T$ we obtain $t(i)=\frac{z(p(i))}{1-z(p(i))} T_{i}$. Thus we can restate (39) in terms of own market share as

$$
\Pi(i)=p(i) V-\Phi\left(i, \frac{z(p(i))}{1-z(p(i))} T_{i}\right)
$$

A Nash equilibrium is a probability vector $(p(1), \ldots, p(n))$ and an aggregate $T>0$ such that $T_{i}=(1-z(p(i))) T$ and $p(i)$ maximizes (39'). It follows that any interior Nash equilibrium satisfies the FOC system

$$
V=\frac{\varphi(i, z(p(i)) T)}{1-z(p(i))} z^{\prime}(p(i)) T
$$

showing that we can apply our rotation tools to study the distributive comparative-statics as in the earlier models. One advantage, however, of the previous contest model is that it yields a slightly more tractable structure. ${ }^{43}$ From (40) it follows that the distributional effects of $d V$

\footnotetext{
${ }^{41}$ Another justification is that with continuum agents $T=\int(t(s) d s$ does not depend on $t(i)$. This type of argument was used by Dixit and Stiglitz (1977) and later by Melitz (2003) in the context of monopolistic competition with continuum firms. Hefti (2016b) shows that similar principles apply to Nash and aggregatetaking behavior in sum-aggregative games with respect to equilibrium uniqueness and stability.

${ }^{42}$ More generally, the following technique can be used in games with a sum-aggregative structure, such as the Cournot model.

${ }^{43}$ The respective FOC is $V=\varphi(i, z(p(i)) T) z^{\prime}(p(i)) T$. For given $p(i), T$ marginal costs are thus higher if the own effect on the aggregate are taken into account. This is intuitive, because an increase in $t(i)$ also increases $T$ which, ceteris paribus, decreases $t(i) / T$.
} 
depend only on the elasticity of the cost function, similar to Proposition 12.

Proposition 15 Let $\pi(t(i), T)$ be a zero-homogeneous CSF. The distributional patterns of $p(i)$ induced by $d V$ are determined by the t-elasticity of marginal costs alone. Specifically, if $\Phi(i, t)=$ $c(i) t^{\eta}$, then $p(i), \Pi(i) / \Pi(j)$ as well as $t(i) / t(j)$ are invariant to $x$.

Evaluated in the special case of a Tullock CSF with arbitrary noise parameter $\eta \geq 1$, Proposition 15 shows that the choice of $V$ has no distributional impacts also in the Nash equilibrium. Proposition 12 is not valid if $V=V(T, x)$ and agents take into account own effects on the aggregate $T$. The reason is that $V(\cdot)$ then depends on $t(i)$. From Proposition 13 we know that an idiosyncratic prize function may induce rotations of $p(i)$. Nevertheless, nothing prevents us from applying the previous methods also in this case. As a final illustration, suppose that $V(T, x)$ is affine-linear in $T$. Then a similar finding as in Proposition 13 results. ${ }^{44}$

Proposition 16 Suppose that $V=\alpha \sum t(s)+\beta, \Phi(i, t)=c(i) t^{\eta}$, where $\beta>0$ and $\pi(x)=x$ (Tullock CSF). If $\alpha \geq 0$, then $d \alpha>0$ induces an $O R$ of $p(i)$. If $\alpha<0$, then $d \beta>0$ induces an OR of $p(i)$.

In the advertising interpretation, Proposition 16 thus suggests that if aggregate advertising has a strong positive externality among the advertisers, we can expect to see very concentrated markets.

\section{Conclusion}

The methods developed in this paper can be used to obtain predictions about the distributional effects of certain exogenous shocks to a model with heterogeneous agents. Our distributional predictions are directly derived in term of output variables, such as (relative) market shares or relative payoffs, rather than by the strategic variables that effectively generated them (e.g., effort levels in contests or prices in monopolistic competition). The advantage is that the former is more likely to be available in empirical data. Obtaining "robust" comparative-static predictions, which do not require to ex ante restrict heterogeneity just to two different types may be particularly valuable to applied work, because real-world agents barely are symmetric but the precise extent of the heterogeneity may be unknown. While we have applied or tools to several different

\footnotetext{
${ }^{44}$ In the following proposition we assume parameters such that payoffs are strictly quasiconcave in $p(i)$.
} 
economic models this is far from being comprehensive, and some of our applications may be worth a detailed separate consideration. ${ }^{45}$

Our approach could provide a valuable instrument for normative questions related to distribution and welfare. A planer may have aim to implement a set of instruments to obtain a certain distributional outcome, e.g., because of fairness concerns or other considerations. While we did not study such questions directly, it is clear that understanding the distributional comparativestatic effects of various possible instruments in a certain economic context constitutes a central milestone in any such attempt.

\section{References}

Amegashie, J. A. (1999). The number of rent-seekers and aggregate rent-seeking expenditures: an unpleasant result. Public Choice, 99(1-2):57-62.

Anderson, S. P., De Palma, A., and Thisse, J. F. (1992). Discrete choice theory of product differentiation. MIT press.

Bagwell, K. (2007). The economic analysis of advertising. In Armstrong, M. and Porter, R., editors, Handbook of Industrial Organization, volume 3. Amsterdan: Elsevier B.V.

Chung, T. Y. (1996). Rent-seeking contest when the prize increases with aggregate efforts. Public Choice, $87: 55-66$.

Clark, D. J. and Riis, C. (1998). Competition over more than one prize. The American Economic Review, 88(1):276-289.

Dixit, A. K. and Stiglitz, J. E. (1977). Monopolistic competition and optimum product diversity. The American Economic Review, 67:297-308.

Ellison, G. and Wolitzky, A. (2012). A search cost model of obfuscation. The RAND Journal of Economics, 43(3):417-441.

Hefti, A. (2016a). Limited attention, competition and welfare. Working paper.

Hefti, A. (2016b). On the relationship between uniqueness and stability in sum-aggregative, symmetric and general differentiable games. Mathematical Social Sciences, 80:83-96.

Hefti, A. and Liu, S. (2016). Targeted advertising and limited attention. Working Paper.

Jackson, M. O. and Yariv, L. (2016). The non-existence of representative agents. Working Paper.

Johnson, J. P. (2013). Targeted advertising and advertising avoidance. The RAND Journal of Economics, 44(1):128-144.

Konrad, K. (2009). Strategy and Dynamics in Contests. Oxford University Press.

Melitz, M. J. (2003). The impact of trade on intra-industry reallocations and aggregate industry productivity. Econometrica, 71(6):1695-1725.

\footnotetext{
${ }^{45}$ Adapting a famous word of Fermat: We believe to have developed a wonderful tool, but the bounds of this article are to tight to contain it.
} 
Posner, R. A. (1992). Economic analysis of law. Boston, Toronto and London: Little, Brown and Company.

Szymanski, S. (2003). The economic design of sporting contests. Journal of economic literature, 41(4):1137-1187.

Topkis, D. M. (1998). Supermodularity and Complementarity. Princeton: University Press. 


\section{A Appendix}

\section{A.1 Continuum representation for atomistic agents}

We now show that the equilibrium distribution in case of $n \in \mathbb{N}$ atomistic ("discrete") agents can be identified with our finite step density model. The following argument considers the case, where heterogeneity enters the model through a cost coefficient function as in (5). This should suffice to make evident that the representation result applies similarly to other cases as well. Consider a population consisting of $n \in \mathbb{N}$ atomistic (or "discrete") agents, indexed by $\{1 / n, 2 / n, \ldots, 1\}$. Suppose that the agents differ in their cost coefficient $c(i), i \in\{1 / n, 2 / n, \ldots, 1\}$. Then, the agents can be partitioned into $1 \leq K \leq n$ groups of identical agents, with group size $n_{k}, \sum_{k} n_{k}=n$. This partition gives $1 \leq K \leq n$ equivalence classes (groups) of sizes $n_{1}, \ldots, n_{K}$, $\sum_{k} n_{k}=n$. We identify each group by a "representative" agent $i_{k}$. In equilibrium every agent $(i / n)$ chooses $p^{d}(i / n)$ ( $d$ for "discrete") to maximize her payoff $\Pi(i)$, where $p^{d}(i / n)$ must satisfy $\sum_{i=1}^{n} p^{d}(i / n)=1$. Let $p(i)$ denote the (step) density function that characterizes our (continuum) equilibrium from definition 1 with the corresponding cost step function $c(i)=c\left(i_{k}\right)$ on $\left[i_{k}, i_{k+1}\right)$, and group measures $\gamma_{1}, \ldots, \gamma_{K}$ satisfying $\gamma_{k}=n_{k} / n$. We now establish the formal equivalence between the discrete equilibrium probability distribution $\left\{p^{d}(1 / n), \ldots, p^{d}(1)\right\}$ and the equilibrium step density $p(i)$.

Theorem 5 (Continuum Representation) Let $n \in \mathbb{N}$ and suppose that agents are partitioned in $K$ cost groups. If $\left\{p^{d}(i / n)\right\}$ corresponds to the discrete equilibrium and $p(i)$ is the equilibrium (step) density of the respective continuum problem, then $p^{d}(i / n)=\frac{1}{n} p(i / n)$ is satisfied for all $i \in\{1, \ldots, n\}$

Proof: In the continuum case we only have to solve the optimization problem for a representative agents $i_{k}$. In the discrete equilibrium $1=\sum_{i=1}^{n} p^{d}(i / n)=\sum_{k=1}^{K} p^{d}\left(i_{k}\right) n_{k}$. The claim now is that $\frac{1}{n} p\left(i_{k}\right)=p^{d}\left(i_{k}\right)$ for $k=1, \ldots, K$. But because in the continuum equilibrium we must have

$$
1=\int_{0}^{1} p(i) d i=\sum_{k=1}^{K} p\left(i_{k}\right) \gamma_{k}=\sum_{k=1}^{K}\left(\frac{1}{n} p\left(i_{k}\right)\right) n_{k}
$$

the claim follows from the uniqueness of equilibrium.

Hence the continuum step-function case and the atomistic case are equivalent up to the multiplicative constant $1 / n$ (independent of group composition), which means that we can work 
with either model, and justifies our procedure of the main text. It then also follows that $p\left(i_{k}\right) \gamma_{k}=p^{d}\left(i_{k}\right) n_{k}$ corresponds to the market share of a member of group $k$, illustrating why we used the notion of a "representative" agent.

Because Theorem 5 remains valid as $n$ grows arbitrarily large, this provides the following justification for using strictly increasing cost coefficient functions (class II) as an approximation for the case of many different agents. Suppose that $c(i)$ is a class II function defined on $[0,1]$ (e.g., $c(i)=1+i$ ), and let $p(i)=p(c(i))$ denote the corresponding equilibrium density (a strictly decreasing, continuous function). Then, because $c(i)$ is continuous on a compact interval, for $n \in \mathbb{N}$ the sequence of step functions defined by $c_{n}(i)=c(i)$ if $i \in\{0,1 / n, 2 / n, \ldots, 1\}$ and $c_{n}(i)=$ $c(s / n)$ for $i \in(s / n,(s+1) / n), s \in\{0,1, \ldots, n-1\}$, converges (uniformly) to $c(i)$ as $n \rightarrow \infty .{ }^{46}$ Consider the atomistic equilibrium distribution $p^{d}(i / n)$ induced by $c(0), c(1 / n), c(2 / n), \ldots, c(1)$.

By Theorem $5, n p^{d}(i / n)=p(i / n)$, where $p(i / n)$ is the step-density version of $p^{d}(i / n)$. More precisely, for a given $n \in \mathbb{N}$ this density is a decreasing finite step function with $p_{n}(i)=p\left(c_{n}(i)\right)$, where $c_{n}(i)$ is as defined above. Because $c_{n}(i) \rightarrow c(i)$ and $p(i)$ is continuous, we have $p(i)=$ $p(c(i))=p\left(\lim c_{n}(i)\right)=\lim p\left(c_{n}(i)\right)=\lim p_{n}(i)$. This shows that while, of course, the atomistic $p^{d}(i / n)$ becomes arbitrarily close to zero as $n$ grows large, the "scaled" distribution law as captured by the step-density version $p(i / n)$ approaches $p(i)$.

\section{A.2 Proofs}

Proof of Theorem 1 The proof consists of two steps. i) Fix $i \in[0,1]$ and $T>0$ arbitrarily. (A1) assures that the equation (4) has a unique solution $p(i ; T)>0$, and that this solution indeed maximizes (3) given $T$. Now, consider the function $p(i, T) \equiv p(i ; T)$, noting that $p(i, \cdot)$ is a strictly decreasing $C^{1}$-function on $(0, \infty)$ as a consequence of the Implicit Function Theorem, the strong quasiconcavity assumption in (A1), and the last assumption of (A2). Moreover, $p(\cdot, T)$ is a decreasing function because of $(\mathrm{A} 3)$ and, hence, $p(\cdot, T)$ is integrable over $[0,1]$, so let $G(T) \equiv \int_{0}^{1} p(i, T) d i$, noting that $G$ is differentiable. ii) We show: $\exists ! T>0: G(T)=1$. Fix $i \in[0,1]$. By (A3) there must exist $T_{i}>0: g\left(i, 1, T_{i}\right)=\varphi\left(i, 1, T_{i}\right)$. Therefore, $\exists T_{0}>0$ such that $p\left(0, T_{0}\right)=1$. Because $p(i, \cdot)$ strictly decreasing, it follows that $p(0 ; T)<1$ for $T>T_{0}$. Since $p(\cdot, T)$ is decreasing, we must have $p(i, T)<1$ for any $i \in[0,1]$ and $T>T_{0}$, which implies that

\footnotetext{
${ }^{46} \mathrm{Such}$ approximations of continuous functions by a sequence of step functions are a standard result in real analysis and integration theory.
} 
$\lim _{T \rightarrow \infty} G(T)<1$. Similarly, it follows that $\exists T_{1}>0$ such that $p\left(1 ; T_{1}\right)=1$. Thus $p\left(i, T_{1}\right)>1$ for $i \in[0,1]$ and $T<T_{1}$, hence $\lim _{T \rightarrow 0} G(T)>1$. As $G(\cdot)$ continuous, $\exists T>0$ such that $G(T)=1$, and uniqueness follows from the fact that, for each $i \in[0,1], p(i ; T)$ and hence $G(T)$ is strictly decreasing in $T$. Finally, $\Pi(i)>0$, because $p(i)=p(i ; T)>0$ is the unique maximizer and $\left.\Pi(i)\right|_{p(i)=0}=0$.

Proof of Corollary 1 To see that $p(i)>(\geq) p(j)$ as claimed, note that $g(i, p, T) \gtreqless \varphi(i, p, T)$ $\Leftrightarrow p(i) \gtreqless p$ because, by strong quasiconcavity (A3), $g(i, \cdot, T)$ must intersect $\varphi(i, \cdot, T)$ from above at $p(i)$ (see Figure 1). Further, in equilibrium

$$
g(i, p(j), T)>(\geq) g(j, p(j), T)=\varphi(j, p(j), T)>(\geq) \varphi(i, p(j), T)
$$

Hence $g(i, p(j), T) \geq \varphi(i, p(j), T)$ and thus $p(i) \geq p(j)$, where these two inequalities are strict if at least one inequality in (41) is strict. It also follows that $p(i)=p(j)$ if all inequalities in (41) are equalities, which proves the last claim of Corollary 1. The claims about payoffs holds because

$$
\begin{aligned}
\Pi(i) & =B(i, p(i), T)-\Phi(i, p(i), T) \\
& \geq B(i, p(j), T)-\Phi(i, p(j), T)>(\geq) B(j, p(j), T)-\Phi(j, p(j), T)=\Pi(j),
\end{aligned}
$$

where the first inequality follows from optimality, and the second type of inequality follows from (A3) and the additional presumption in Corollary 1.

Proof of Proposition 1 Define $g(i) \equiv p\left(i, x^{\prime}\right)-p(i, x)$, and note that $\int g(s) d s=0$. Suppose that $g(0) \leq 0$ By presupposition, $g$ is decreasing, right-continuous and, by SSD, $\exists i_{0} \in(0,1)$ : $0 \geq g(0)>g(i), \forall i \geq i_{0}$. Hence $\int g(s) d s<0$, a contradiction. Therefore $g(0)>0$, and a similar argument shows that $g(1)<0$. Because $g$ is decreasing, right-continuous and $g(0)>0$, the set $\{i: g(i)>0, i>0\}$ is non-empty, and we let $i_{0}=\sup \{i>0: g(i)>0\}$, noting that $i_{0} \in(0,1)$. It follows that $p\left(i, x^{\prime}\right)>p(i, x)$ on $\left(0, i_{0}\right)$, and $\int_{0}^{i_{0}} g(s) d s>0$. Because $g$ decreases and $\int g(s) d s=0$, the set $\left\{i: g(i)<0, i \geq i_{0}\right\}$ is non-empty, and we set $i_{1}=\inf \left\{i \geq i_{0}: g(i)<0\right\}$. If $i_{0}<i_{1}$ then $g(i)=0$ on $\left(i_{0}, i_{1}\right)$, as $g$ is decreasing and right-continuous. These facts together imply that $p\left(\cdot, x^{\prime}\right)$ is $\mathrm{OR}$ of $p(\cdot, x)$. 
Proof of Proposition 2 Define $g(i) \equiv \frac{p\left(i, x^{\prime}\right)}{p(i, x)}$, and establish $g(0)>1, g(1)<1$ and the existence of $0<i_{0} \leq i_{1}<1$ such that $g(i)>1$ if $i<i_{0}, g(i)=1$ if $i \in\left[i_{0}, i_{1}\right)$ and $g(i)=1$ for $i \geq i_{1}$ by exactly the same reasoning as in the proof of Proposition 1 .

Proof of Corollary 2 We only show the OR case. Define $f(x ; i, j) \equiv \frac{p(i, x)}{p(j, x)}$. If $p(\cdot)$ is class II and (11) is satisfied, then $f(x ; i, j)>f\left(x_{0} ; i, j\right)$ whenever $x>x_{0}$, and the claim follows from Proposition 2. If $p(\cdot)$ is class I, then $p(\cdot, x)$ is piecewise constant for any given $x \in X$, with a finite number of downward jumps. If (11) is satisfied for any two $i, j \in(0,1)$ with $j \triangleright i$ that are not jump points of $p(\cdot, x)$, we must have that $f(x ; i, j)>f\left(x_{0} ; i, j\right)$ for any such $i, j$ and any $x>x_{0}$, proving the claim by Proposition 2 .

Proof of Lemma 1 By (A1), equation (4) has a unique solution for given $T$, $x$, denoted by $p(i ; T, x)$. Define $G(T ; x) \equiv \int p(i ; T, x) d i$, note that in equilibrium $G(T ; x)=1$. Quasiconcavity (A1) and $g_{x}>0$ imply that $p(i ; T, x)$ is strictly increasing in $x$ for a fixed $T$ and any $i \in[0,1]$. Because, by $(\mathrm{A} 2), G_{T}(T ; x)<0$ (see the proof of Theorem 1), applying the Implicit Function Theorem to the equilibrium equation $G(T(x), x)=1$ yields $T^{\prime}(x)>0$.

Proof of Theorem 2 We proof the first claim by contradiction. Hence suppose that $R=0$ $\forall i, j \in[0,1]$ and any $x \in X$, but $\exists j \in(0,1)$ such that $\Delta_{j} \neq 0$ (and hence $d p(j) \neq 0$ ). Because in equilibrium the integral condition

$$
\int \frac{\partial p(s, x)}{\partial x} d s=0
$$

must hold, we can suppose, wlog, that $\Delta_{j}>0$ for some $j \in(0,1)$. By (14) we must have $\Delta_{i}>0$ for all $i<j$, and because of (42) $\exists j^{\prime} \in(0,1), j^{\prime}>j$, such that $\Delta_{i}<0$ for all $i>j^{\prime}$. Take $i<j$ and $i^{\prime}>j^{\prime}$. Then $\Delta_{i}>0$ but also $\Delta_{i}=k \Delta_{i^{\prime}}<0$, contradiction. Turning to the second claim, note that if $R \neq 0$ for some $i, j$ then $\Delta_{i}=0 \forall i \in[0,1]$ is impossible by (14). Hence $\forall x \in X$ $\exists i$ : $\Delta_{i}(x) \neq 0$, or equivalently $\frac{\partial p(i, x)}{\partial x} \neq 0$, and therefore $\exists \delta>0$ such that $p\left(i, x^{\prime}\right) \neq p(i, x)$ for $x^{\prime} \in(x-\delta, x+\delta)$, thus we have $p\left(\cdot, x^{\prime}\right) \neq p(\cdot, x)$ on that interval. 
Proof of Theorem 3 Step 1: We first prove the second claim, and restrict attention to the OR-case (the IR-case is similar). Because $R\left(x_{0}\right)$ is uniformly positive, $\exists i \in(0,1): \Delta_{i}\left(x_{0}\right)>0$ by the proof of Theorem 2. By the integral condition (42), there then must also be $i^{\prime} \in(0,1)$ : $\Delta_{i^{\prime}}\left(x_{0}\right)<0$. It then follows from (14) that $i_{0}=\sup \left\{i \in[0,1]: \Delta_{i}\left(x_{0}\right)>0\right\} \in(0,1), i_{1}=\inf \{i \in$ $\left.[0,1]: \Delta_{i}\left(x_{0}\right)<0\right\} \in(0,1)$ and $i_{0} \leq i_{1}$. For any $i<i_{0}: \Delta_{i}\left(x_{0}\right)>0$ and hence $\frac{\partial p\left(i, x_{0}\right)}{\partial x}>0$. This derivative condition implies that $\forall i<i_{0} \exists \delta_{i}>0: p(i, x)>p\left(i, x_{0}\right) \forall x \in\left(x_{0}, x_{0}+\delta_{i}\right)$.

Step 2: Because $p(\cdot, x)$ is class I, there is a finite number of equivalence classes to the left of $i_{0}$, and we only need to consider a single $i$, with corresponding $\delta_{i}$, for each step of $p(\cdot, x)$ to the left of $i_{0}$. Let $\delta^{0}>0$ be the smallest value of these $\delta_{i}$. We have thus shown that $\exists i_{0} \in(0,1)$ such that for any given $x \in\left(x_{0}, x_{0}+\delta^{0}\right)$ we have $p(i, x)>p\left(i, x_{0}\right)$ for $i<i_{0}$. A similar argument shows that we can find $\delta^{1}>0$ such that $\exists i_{1} \in(0,1)$ such that $p(i, x)<p\left(i, x_{0}\right)$ for $i>i_{1}$ and any $x \in\left(x_{0}, x_{0}+\delta^{1}\right)$. Let $\delta \equiv \min \left\{\delta^{0}, \delta^{1}\right\}>0$. Summarizing, the arguments so far show that $\exists i_{0}, i_{1} \in(0,1), i_{0} \leq i_{1}$ such that for $x \in\left(x_{0}, x_{0}+\delta\right)$ we have $p(i, x)>p\left(i, x_{0}\right)$ for $i<i_{0}$ and $p(i, x)<p\left(i, x_{0}\right)$ for $i>i_{1}$. If $\Delta_{i} \neq 0$ for any $i \in\left(i_{0}, i_{1}\right]$ we must have $i_{0}=i_{1}$ and the proof is complete. Now suppose that $\exists m \in\left(i_{0}, i_{1}\right]: \Delta_{m}\left(x_{0}\right)=0$. Then (14) implies that $\Delta_{i}>0$ for any $m \triangleright i$, and $\Delta_{j}<0$ for any $j \triangleright m$. But this shows that there can be at most one step of $p(\cdot, x)$ for which $\Delta_{m}\left(x_{0}\right)=0$. It follows that independent of whether $p\left(m, x^{\prime}\right)>=<p(m, x)$ for $x \in\left(x_{0}, x_{0}+\delta\right), p\left(\cdot, x^{\prime}\right)$ must be OR of $p\left(\cdot, x_{0}\right)$. We now prove the first claim. By step 1 and the global uniform positivity of $R$, we must have $\Delta_{0}(x)>0$ and thus $\frac{\partial p(0, x)}{\partial x}>0$ for any $x>x_{0}$ (note that this result is valid also if $p(\cdot)$ is of class II), hence $p\left(i, x^{\prime}\right)>p\left(i, x_{0}\right) \forall i \in[0]$. Similarly, $\Delta_{1}(x)<0$ for all $x>x_{0}$, hence $p\left(i, x^{\prime}\right)<p\left(i, x_{0}\right) \forall i \in[1]$.

Proof of Corollary 3 We only prove the first claim as the remaining claims are proved identically. Recall from the equivalence class argument in step 2 of the proof of Theorem 3 that there is a finite number of $\Delta_{i}\left(x_{0}\right)>0$, possibly a single $\Delta_{m}\left(x_{0}\right)=0$ and a finite number $\Delta_{j}\left(x_{0}\right)<0$. Define $f(i, j, x)=\frac{p(i, x)}{p(j, x)}$. If $\Delta_{i^{\prime}}\left(x_{0}\right) \geq 0$ then any $i$ with $i^{\prime} \triangleright i$ has $\Delta_{i}\left(x_{0}\right)>\Delta_{i^{\prime}}\left(x_{0}\right)$ by (14). Hence we must have $\frac{\partial f\left(i, i^{\prime}, x_{0}\right)}{\partial x}>0$. If $\Delta_{i^{\prime}}\left(x_{0}\right)<0$ but $\Delta_{i}\left(x_{0}\right)>0$, then obviously $\frac{\partial f\left(i, i^{\prime}, x_{0}\right)}{\partial x}>0$ Thus for any pair $\left(i, i^{\prime}\right)$ as described above $\exists \delta_{i, i^{\prime}}>0$ such that $f\left(i, i^{\prime}, x^{\prime}\right)>f\left(i, i^{\prime}, x_{0}\right)$ for all $x^{\prime} \in\left(x_{0}, x_{0}+\delta_{i, i^{\prime}}\right)$. The proof is completed by letting $\delta>0$ be the smallest among these (finitely many) $\delta_{i, i^{\prime}}$ and $\delta^{0}, \delta^{1}$ as identified in the proof of Theorem 3. 
The claim follows immediately from Corollary 2 because, by (14), if $R(x)$ is globally uniformly positive (negative) and $k=1$, then $\Delta_{i}(x)>(<) \Delta_{j}(x)$, for any $j \triangleright i$ and any $x \in X$, and hence condition (11') holds.

Proof of Proposition 4 We only prove the uniformly positive case (the negative case is established under the same type of arguments). We need to show that for

$$
A(i)=\frac{g_{T}(i)}{g(i)} T^{\prime}(x)+\frac{g_{x}(i)}{g(i)}
$$

we have $A(i)>A(j)$ whenever $j \triangleright i$. So take any $j \triangleright i$. First, $h_{T}\left(i, p^{\prime}, T, x_{0}\right) \geq h_{T}\left(i, p, T, x_{0}\right)$ and $h_{T}\left(i, p, T, x_{0}\right) \geq h_{T}\left(j, p, T, x_{0}\right)$ yield

$$
h_{T}\left(i, p(i), T, x_{0}\right) \geq h_{T}\left(i, p(j), T, x_{0}\right) \geq h_{T}\left(j, p(j), T, x_{0}\right)
$$

and because $T^{\prime}(x)>0$ by Lemma 1 we have

$$
\frac{g_{T}(i)}{g(i)} T^{\prime}(x) \geq \frac{g_{T}(j)}{g(j)} T^{\prime}(x)
$$

where the inequality is strict, whenever at least one of the initial inequalities is strict. Second, $h_{x}\left(i, p, T, x_{0}\right) \geq h_{x}\left(j, p, T, x_{0}\right)$ and $h_{x}\left(i, p^{\prime}, T, x_{0}\right) \geq h_{x}\left(i, p, T, x_{0}\right)$ yield

$$
h_{x}\left(j, p(j), T, x_{0}\right) \leq h_{x}\left(j, p(i), T, x_{0}\right) \leq h_{x}\left(i, p(i), T, x_{0}\right)
$$

and hence also

$$
\frac{g_{x}(i)}{g(i)} \geq \frac{g_{x}(j)}{g(j)}
$$

where, again, the inequality is strict if one of the previous inequalities is strict. This shows that $R\left(x_{0}\right)>0$, and the global case follows immediately. 
Proof of Proposition 5 Consider the first two rows of Table 1 . If $\gamma_{i}=\gamma \geq 1, \forall i \in[0,1]$, then (22) implies

$$
\frac{p(i)}{p(j)}=\left(\frac{c(j)}{c(i)}\right)^{\frac{\eta-1}{\eta(\gamma-1)+1}}\left(\frac{r(i)}{r(j)}\right)^{\frac{\gamma \eta}{\eta(\gamma-1)+1}}
$$

from which $\frac{\partial}{\partial \eta} \frac{p(i)}{p(j)}>0$ and $\frac{\partial}{\partial \gamma} \frac{p(i)}{p(j)}<0$, and the first column in Table 1 follows from Corollary 2 . The third and fifth columns follow from $\Pi(i)=p(i) I\left(\frac{\gamma \eta-\eta+1}{\gamma \eta}\right)$, because then $\frac{\Pi(i)}{\Pi(j)}=\frac{p(i)}{p(j)}$. From $p(i)=\frac{r_{i}^{\eta} P_{i}^{1-\eta}}{T}$ we obtain

$$
\frac{P(i)}{P(j)}=\left(\frac{c(i)}{c(j)}\right)^{\frac{1}{1+(\gamma-1) \eta}}\left(\frac{r(i)}{r(j)}\right)^{\frac{(\gamma-1) \eta}{1+(\gamma-1) \eta}}
$$

and the fourth column follows from differentiating this expression. The remaining two rows of Table 1 are obvious from the above derivations.

Proof of Proposition 6 The fact that $T^{\prime}(I)<0$ gives $\frac{\partial q_{i}}{\partial I}>0 \forall i \in[0,1]$ by the left equation in (23). The right equation in (23) implies that

$$
\frac{q_{i}^{\left(\gamma_{i}-1\right)+\frac{1}{\eta}}}{q_{j}^{\left(\gamma_{j}-1\right)+\frac{1}{\eta}}}
$$

is independent of $I$. Because $q(\cdot) \geq 1$ and $\gamma_{i}<\gamma_{j}, j \triangleright i$, this together with $d q_{i}, d q_{j}>0$ further implies that $\frac{q_{i}}{q_{j}}$ is strictly increasing in $I$. Therefore $\frac{p(i)}{p(j)}$ increases in $I$ by $\left(20^{\prime}\right)$ which, by Corollary 2 , implies an OR of $p(\cdot)$. Since $\Pi(i)=p(i) I\left(\frac{\gamma_{i} \eta-\eta+1}{\gamma_{i} \eta}\right)$, relative profits increase in $I$, and because $p(i)=\frac{r_{i}^{\eta} P_{i}^{1-\eta}}{T}$ so do relative prices $\frac{P_{j}}{P_{i}}$.

Proof of Proposition 7 By FOC, $P(T, x)=\varphi(i, q(i))$, either $d q(i)>0$ or $d q(i) \leq 0 \forall i$. Then, $d x>0$ implies that $q(i)$ increases; if $d q(i) \leq 0 \forall i$, then $d P \leq 0$ by FOC but because also $d T \leq 0$, $P_{T}<0$ and $P_{x}>0$ this is impossible. A standard Envelope-theorem argument shows that $\Pi(i)$ increases in $x$. If $\Phi(i, q)$ is a power function with common and constant exponent $\eta$, so is $\phi(i, q)$, $R_{i j}=0$ by $(29)$ and $p(i)$ is invariant to $x$ by Theorem 2 . If $\Phi(i, q)$ is a power function for each $i$, then $\Pi(i)=P(T, x) p(i) T \frac{\eta_{i}-1}{\eta_{i}}$. Hence if $\eta_{i}=\eta \forall i$, then $x$ does not affect relative profits and quantities as long as $p(i)$ does not change. Profit and quantity differences increase in $x$ by the remark following Proposition 2. Let $\Phi(i, q)=q^{\eta(i)}$ as stated by the proposition. Because $P(T(x), x)>\eta(1)$, we must have $q(i)>1$ by the FOC, $\forall i$, and because also $\eta(i) q(i)^{\eta(i)-1}=$ 
$\eta(j) q(j)^{\eta(j)-1}$, we must have $q(i)>q(j)$ and hence $p(i)>p(j)$ for any $j \triangleright i$. Because $T^{\prime}(x)>0$, $\operatorname{sign}(A(i)-A(j))=(\eta(j)-\eta(i))>0$ by $(29)$, hence an OR results, and the claims about relative profits and quantities follows immediately.

Proof of Proposition 8 Since $T^{\prime}(x)>0$, we have $\operatorname{sign} R_{i j}=\operatorname{sign}(A(i)-A(j))=\operatorname{sign}\left(\frac{\varphi_{T}(j)}{\varphi(j)}-\frac{\varphi_{T}(i)}{\varphi(i)}\right)$, where where

$$
\frac{\varphi_{T}(i)}{\varphi(i)}=\frac{\sum s(s-1) a_{s} p(i)^{s-1} T^{s-2}}{\sum s a_{s}(p(i) T)^{s-1}} .
$$

Then

$$
\frac{\partial}{\partial p}\left(\frac{\varphi_{T}(i)}{\varphi(i)}\right)>0 \Leftrightarrow \sum s(s-1)^{2} a_{s} x^{s-1} \sum s a_{s} x^{s-1}>\left(\sum s(s-1) a_{s} x^{s-1}\right)^{2}
$$

We claim that the second inequality holds. Note that both sides of this inequality are polynomials of degree $2(m-1)$, hence it is of the form

$$
u_{1} x+u_{2} x^{2}+\ldots+u_{2(m-1)} x^{2(m-1)}>w_{1} x+w_{2} x^{2}+\ldots+w_{2(m-1)} x^{2(m-1)}
$$

We now claim that $u_{k} \geq w_{k}, k=1, \ldots, m-1$, where the inequality is strict for some $k$. Let $s, s^{\prime} \in\{2, \ldots, m\}$ be such that $s+s^{\prime}=k$ for a given $k$. Then $A=s(s-1)^{2} a_{s} s^{\prime} a_{s^{\prime}}+s^{\prime}\left(s^{\prime}-1\right)^{2} a_{s^{\prime}} s a_{s}$ is a summand in the calculation of $u_{k}$, while $B=2 s(s-1) a_{s} s^{\prime}\left(s^{\prime}-1\right) a_{s^{\prime}}$ is the corresponding summand in the calculation of $w_{k}$. If $a_{s}=0$ or $a_{s^{\prime}}=0$, then $A=B$, so let $a_{s}, a_{s^{\prime}}>0$. If $s=s^{\prime}$ then again $A=B$, so let $s \neq s^{\prime}$ (such $s, s^{\prime}$ exist by presumption). Claim: $A>B$. To see this is suffices to show that $s(s-1)^{2} s^{\prime}+s^{\prime}\left(s^{\prime}-1\right)^{2} s>2 s(s-1) s^{\prime}\left(s^{\prime}-1\right)$, which is equivalent to $(s-1)^{2}-2(s-1)\left(s^{\prime}-1\right)+\left(s^{\prime}-1\right)^{2}>0$. Since $s, s^{\prime}>1$ the last inequality is satisfied. Because $p(i)>p(j)$ for any $j \triangleright i$, this implies that $\frac{\varphi_{T}(i)}{\varphi(i)}>\frac{\varphi_{T}(j)}{\varphi(j)}$, hence $R_{i j}<0$, showing that an IR results.

Proof of Proposition 9 Aggregation of firm FOC yields $T(P)=\int \varphi^{-1}(i, P) d i$, and $T^{\prime}(P)>$ 0 because $\varphi_{q}(i, q)>0 . T^{d}=T$ and aggregate demand imply that $P T(P)=\omega+\Pi(P)$, from which $P^{\prime}(\omega)=\left(T+P T^{\prime}(P)-\Pi^{\prime}(P)\right)^{-1}$. Hotelling's Lemma gives $\Pi^{\prime}(P)=\int \partial_{P} \Pi(i) d i=T$. Therefore $P^{\prime}(\omega)>0$, and $T^{\prime}(\omega)>0$ follows. Then, $\partial_{\omega} q(i)>0$ because, by firm FOC and $\varphi_{q}(i, q)>0, \partial_{P} q(i)>0$, and $\partial_{\omega} \Pi(i)>0$ because $\partial_{P} \Pi(i)=q(i)>0$. The remainder of the claim follows from Theorem 3 because $T^{\prime}(\omega)>0$ and $A(i)$ is given by (29). 
Proof of Proposition 10 Note first that $p^{d}\left(i_{c}\right)$ has the monotone ratio property because (32) is linear in $p^{d}\left(i_{c}\right)$, and therefore an OR (IR) of $p^{d}\left(i_{c}\right)$ always implies that $q\left(i_{c}\right) / q\left(j_{c}\right)$ increases (decreases). Equation (32) implies that for $j_{c} \triangleright i_{c}$ the ratio $p^{d}\left(i_{c}\right) / p^{d}\left(j_{c}\right)$ increases (decreases) in $\omega$ if

$$
\frac{d \omega\left(i_{c}\right)+s\left(i_{c}\right) d \Pi}{\omega\left(i_{c}\right)+s\left(i_{c}\right) \Pi}>(<) \frac{d \omega\left(j_{c}\right)+s\left(j_{c}\right) d \Pi}{\omega\left(j_{c}\right)+s\left(j_{c}\right) \Pi}
$$

(i) If $s\left(i_{c}\right)=1 \forall i_{c}$ is used in (43) together with $d \Pi=\Pi^{\prime}(\omega)>0$, we obtain that $p^{d}\left(i_{c}\right) / p^{d}\left(j_{c}\right)$ decreases in $\omega$. Therefore $d \omega>(<) 0$ induces a global IR (OR) of $p^{d}\left(i_{c}\right)$ by Theorem 4 . (ii) Using $\omega\left(i_{c}\right)=\omega \forall i_{c}$ in $(43)$ shows that $p^{d}\left(i_{c}\right) / p^{d}\left(j_{c}\right)$ decreases (increases) in $\omega$ if $\Pi-\Pi^{\prime}(\omega) \omega>(<) 0$. Because $\Pi^{\prime}(\omega)>0$ and $\Pi(0)=0, \Pi-\Pi^{\prime}(\omega) \omega>(<) 0$ if $\Pi(\omega)$ is strictly concave (convex), and the claim follows from Theorem 4 .

Proof of Corollary 4 With iso-elastic costs, $\Pi(i)=P q(i) \frac{\eta-1}{\eta}$, and thus $\Pi=P T \frac{\eta-1}{\eta}$. Together with aggregated consumer FOC, this implies that $P T=\eta \omega$ and $\Pi=(\eta-1) \omega$. Because $\Pi$ is linear in $\omega, p^{d}\left(i_{c}\right)$ and $q\left(i_{c}\right) / q\left(j_{c}\right)$ must be invariant to $\omega$ by the proof of Proposition 10 (ii). Aggregated firm FOC together with $P T=\eta \omega$ imply that $\omega / P=\gamma \omega^{1 / \eta}$, where $\gamma>0$ is a constant. Because $q(i)=\omega / P\left(1+s\left(i_{c}\right)(\eta-1)\right)$ it follows that $\partial_{\omega} q(i)>0$. Hence the facts that $q\left(i_{c}\right) / q\left(j_{c}\right)$ is constant but $q\left(i_{c}\right)>q\left(j_{c}\right)$ both decrease, imply the last claim.

Proof of Proposition 11 Invariance of $p(i)$ and $\frac{\Pi(i)}{\Pi(j)}$ to $\alpha$ and a common $d c(i)$ follow from the constant and common elasticity of costs as argued in the main text. Equilibrium profits are $\Pi(i)=p(i) T \frac{\eta}{\eta-1}$. In equilibrium $T=T^{d}$, and summation of consumer FOC yields $T=\alpha(w+\Pi)$. Using $\Pi=\int \Pi(i) d i$ in this equation implies that

$$
T=\frac{\alpha \eta}{\alpha+(1-\alpha) \eta} w
$$

But because $S\left(i_{c}\right)=s\left(i_{c}\right) \Pi$, capital incomes change in equal proportions, and

$$
\frac{d S\left(i_{c}\right)}{S\left(i_{c}\right)}=\frac{d \Pi(i)}{\Pi(i)}=\frac{d T}{T}
$$

(i) Equation (44) additionally implies that $d T / T=d w / w$. Therefore

$$
\frac{d w+d S\left(i_{c}\right)}{w+S\left(i_{c}\right)}=\frac{d w+d S\left(j_{c}\right)}{w+S\left(j_{c}\right)}
$$


showing that $p\left(i_{c}\right) / p\left(j_{c}\right)$ and $q\left(i_{c}\right) / q\left(j_{c}\right)$ remain constant, proving that $p\left(i_{c}\right)$ is unaffected. Because $f\left(i_{c}\right)=(1-\alpha)\left(1+S\left(i_{c}\right) / w\right)$, also $f\left(i_{c}\right)$ remains constant. Integration of the FOC of the firm problem yields

$$
T=\left(\frac{1}{w \eta}\right)^{\frac{1}{\eta-1}} C, \quad C \equiv \int c(i)^{\frac{1}{1-\eta}} d i .
$$

The aggregate effect of the exogenous common efficiency increase thus is entirely captured by $d C>0$, and (44), (45) together imply that $T^{\prime}(C), w^{\prime}(C)>0$, which further assure that $d \Pi(i)>0$ and $d q\left(i_{c}\right)>0$ for each firm and consumer, respectively. Finally, $d q\left(i_{c}\right)>d q\left(j_{c}\right)$ and $d \Pi(i)>$ $d \Pi(j)$ follow from the above results and the remark after Proposition 2. (ii) Equations (44), (45) then imply that $w^{\prime}(\alpha)<0$ and $T^{\prime}(\alpha)>0$. By the latter also $\partial_{\alpha} \Pi(i)>0$, and hence $\Pi^{\prime}(\alpha)>0$ as well as $d S(i)>0$ (provided that $s(i)>0$ ). Because aggregate output increases so does aggregate labor supply. To prove the OR of $p^{d}\left(i_{c}\right)$, it suffices to show that $p^{d}\left(i_{c}\right) / p^{d}\left(j_{c}\right)$ increases in $\alpha$ by Corollary 2, hence that

$$
\frac{d w+d S\left(i_{c}\right)}{w+S\left(i_{c}\right)}>\frac{d w+d S\left(j_{c}\right)}{w+S\left(j_{c}\right)}
$$

This inequality holds by the fact that $S\left(i_{c}\right)=s\left(i_{c}\right) \Pi, d w<0$ and $d \Pi>0$. The monotone ratio property follows because the consumer FOC is linear in $p^{d}\left(i_{c}\right)$, which immediately also implies that $q\left(i_{c}\right) / q\left(j_{c}\right)$ and $f\left(i_{c}\right) / f\left(j_{c}\right)$ increases. It follows that $d q\left(i_{c}\right)>d q\left(j_{c}\right)$ if $d q\left(i_{c}\right)>0$.

Proof of Proposition 12 The FOC of (34'), evaluated for the case $V(T, x)$, is $V(T, x)=$ $\varphi\left(i, \hat{\pi}^{-1}(p(i)) T\right)$. As $V_{x}>0$ also $T^{\prime}(x)>0$ by Lemma 1 and, using $t(i)=\hat{\pi}^{-1}(p(i)) T$, thus

$$
\operatorname{sign}\left(R_{i j}\right)=\operatorname{sign}\left(\frac{\varphi_{t}(j, t(j)) t(j)}{\varphi(j, t(j))}-\frac{\varphi_{t}(i, t(i)) t(i)}{\varphi(i, t(i))}\right)
$$

which shows the first claim. The second claim then follows, because $R_{i j}=0$ if all $\Phi(\cdot)$ are common-elastic power functions and $\Pi(i)=V(T, x) \frac{\eta p(i) z^{\prime}(p(i))-z(p(i))}{\eta z^{\prime}(p(i))}$.

Proof of Corollary 5 The FOC imply

$$
\frac{p(i)}{p(j)}=\left(\frac{c(j)}{c(i)}\right)^{\frac{1}{\eta-1}}
$$


Thus $d \eta>0$ induces a global IR with monotone ratios by Corollary 2, (18) and Theorem 4, and the claims on relative payoffs and efforts follow from $\Pi(i)=p(i) V(T) \frac{\eta-1}{\eta}$ and $t(i)=p(i) / T$.

Proof of Proposition 13 The FOC are $\alpha T\left(z(p(i))+p(i) z^{\prime}(p(i))\right)+\beta=\eta c(i) z(p(i))^{\eta-1} T^{\eta}$. Evaluation of the conditions in Proposition 4 for $h=\operatorname{Ln}\left(\alpha T\left(z(p)+p z^{\prime}(p)\right)+\beta\right)$ and $x=\alpha$ shows that both $h_{\alpha}(i)>(<) h_{\alpha}(j)$ and $h_{T}(i)>(<) h_{T}(j)$ both are equivalent to $z(p(i))+$ $p(i) z^{\prime}(p(i))>(<) z(p(j))+p(j) z^{\prime}(p(j))$. Thus, if $\frac{z^{\prime \prime}(p) p}{z^{\prime}(p)}>(<)-2 \forall p>0$, such that $z(p)+p z^{\prime}(p)$ is a strictly increasing (decreasing) function, we have from Proposition 4 that $R$ is uniformly positive (negative), and the first claim follows from Theorem 3. Similarly, if $x=\beta$, then $h_{\beta}(i)>h_{\beta}(j)$ if either $\alpha>0$ and $\frac{z^{\prime \prime}(p) p}{z^{\prime}(p)}<-2 \forall p>0$ or if $\alpha<0$ and $\frac{z^{\prime \prime}(p) p}{z^{\prime}(p)}>-2 \forall p>0$. In both cases an OR results, ${ }^{47}$ proving the second claim.

Proof of Proposition 14 Totally differentiating the LHS of (38) yields $d V_{1}+(1-2 p(i)) d V_{2}$. By Proposition 4 the differential change $d V_{1}, d V_{2}$ causes an OR of $p(i)$ if

$$
z(p) \equiv \frac{d V_{1}+(1-2 p) d V_{2}}{V_{1}+(1-2 p) V_{2}}
$$

is increasing in $p$. The claim then follows from $\operatorname{sign}\left(z^{\prime}(p)\right)=\operatorname{sign}\left(d V_{1} V_{2}-d V_{2} V_{1}\right)$.

Proof of Proposition 15 Like in the proof of Proposition 12 we obtain that

$$
\operatorname{sign}\left(R_{i j}\right)=\operatorname{sign}\left(\frac{\varphi_{t}(j, z(p(j)) T) z(p(j)) T}{\varphi(j, z(p(j)) T)}-\frac{\varphi_{t}(i, z(p(i)) T) z(p(i)) T}{\varphi(i, z(p(i)) T)}\right)
$$

proving the first claim. With iso-elastic costs $\varphi\left(i, z(p(i) T)=\eta c(i) z(p(i))^{\eta-1} T^{\eta-1}\right.$ from which $R_{i j}=0$ follows. Hence $p(i)$ is invariant to $d V$, and the remaining claims follow from

$$
\Pi(i)=V \frac{\eta p(i) z^{\prime}(p(i))-z(p(i))(1-z(p(i))}{\eta z^{\prime}(p(i))}
$$

and $t(i) / t(j)=z(p(i)) / z(p(j))$.

\footnotetext{
${ }^{47}$ The cases $\alpha>(<) 0$ and $\frac{z^{\prime \prime}(p) p}{z^{\prime}(p)}>(<)-2$ cannot be signed unambiguously.
} 
Proof of Proposition 16 Rewriting the payoff with the market share variable $p(i)$ gives

$$
\Pi(i)=p(i)\left(\frac{\alpha T_{i}}{1-p(i)}+\beta\right)-c(i)\left(\frac{p(i)}{1-p(i)}\right)^{\eta} T_{i}^{\eta}
$$

from which the equilibrium FOC $\alpha T+\beta(1-p(i))=\eta c(i) p(i)^{\eta-1} T^{\eta}$ can be deduced. For $h(i) \equiv$ $\operatorname{Ln}(\alpha(1-p(i))+\beta)$ we obtain $\operatorname{sign}\left(h_{T}(i)-h_{T}(j)\right)=\operatorname{sign} \alpha, h_{\alpha}(i)>h_{\alpha}(j)$ and $\operatorname{sign}\left(h_{\beta}(j)-\right.$ $\left.h_{\beta}(i)\right)=\operatorname{sign} \alpha$. All claims then follow from Proposition 4. 